
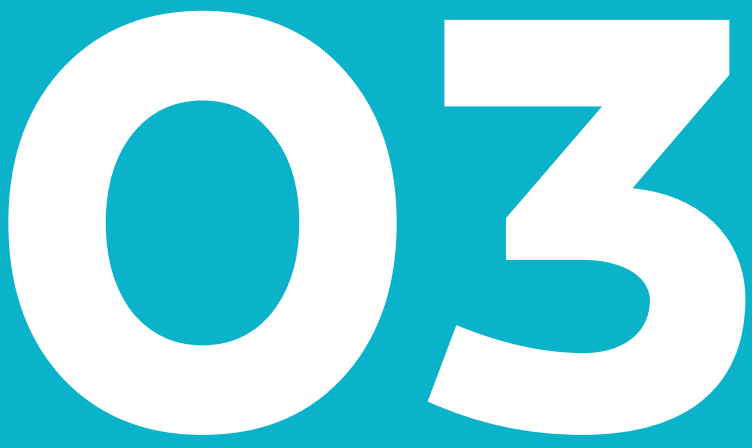

\title{
Estructura de capital, un estudio sobre la producción científica y su contextualización
}

Revisión aplicada sobre las publicaciones existentes en la base de artículos científicos Scopus

Capital structure, a study on scientific production and its contextualization

Applied review of existing publications in the Scopus scientific articles base

Fecha de recepción: 15/01/2020

Fecha de aprobación: 27/08/2020 


\section{Resumen}

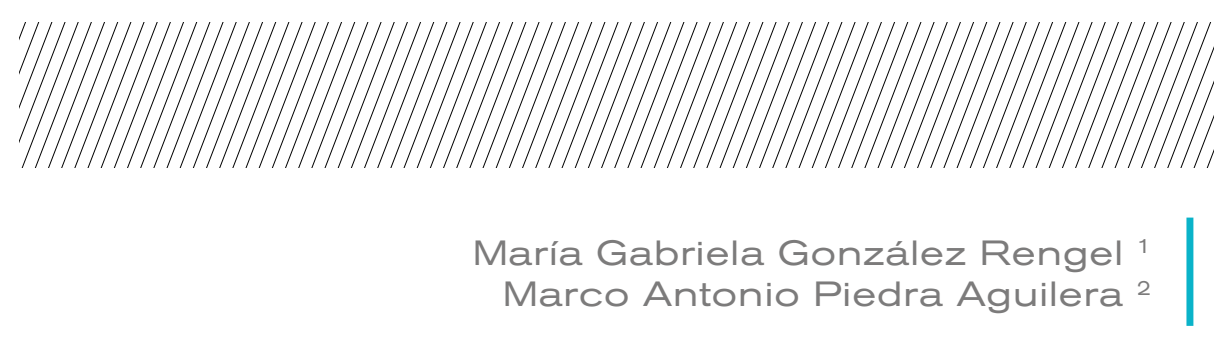

El estudio bibliométrico tiene como propósito analizar el número de publicaciones científicas relacionadas a estructura de capital que se encuentran en la base de datos bibliográficos Scopus, la que permite conocer la cantidad de autores, publicaciones y citas recibidas por cada artículo. Para el análisis se consideró los periodos del 2013 al 2018 a nivel mundial; se obtuvieron 1.429 documentos concernientes a capital estructure según Subject área: Economics, econometrics and Finance $48 \%$ y Business, Management and Accounting 34,40\%. Las áreas de Social Sciences 4.5\% y Decisión Sciences 3.10\%. La tasa de colaboración en investigaciones fue del $78 \%$ y de forma individual el 22\%, Mathematics, Engineering, Environmental, entre otras representan el 10\%. En revistas existen 1.237 artículos, 53 publicaciones en prensa, capítulos de libros 33, documentos de sesión 47 publicaciones, en libros $33 \mathrm{y}$, en cantidades menores, otros tipos de revisiones que representan una cantidad mínima sobre el tema considerando el aporte de la ciencia en las empresas. De la producción científica revisada, la participación de estudios con datos de las empresas ecuatorianas es casi nulo, la única publicación encontrada pertenece a Revelo, Rodríguez \& Mantilla (2017).

Palabras clave:

artículos, empresas, estructura de capital, publicaciones.

Universidad Del Azuay mgabriela.gonzalez@uazuay. edu.ec Universidad Del Azuay mapiedra@uazuay.edu.ec 


\section{ABSTRACT}

$T$

he purpose of the bibliometric study is to analyze the number of scientific publications related to capital structure found in the Scopus bibliographic database, which allows to know the number of authors, publications, citations received for each article. For the analysis, the periods 2013 to 2018 were considered worldwide, 1,429 documents were obtained concerning capital structure according to Subject area: Economics, Econometrics and Finance $48 \%$ and Business, Management and Accounting $34.40 \%$ Social Sciences areas $4.5 \%$ and Decision Sciences $3.10 \%$. The rate of collaboration in research was $78 \%$ and individually 22\%, Mathematics, Engineering and Environmental, among others represent 10\%. In journals there are 1,237 articles, 53 publications in the press, chapters of books 33, session documents 47 publications, in books 33 and in minor quantities other types of reviews that represent a minimum amount on the subject considering the contribution of science in companies. Of the scientific production reviewed, the participation of studies with data from Ecuadorian companies is almost nil, the only publication found belongs to Revelo, Rodríguez \& Mantilla (2017).

Keywords:

articles, companies, capital structure, publications.

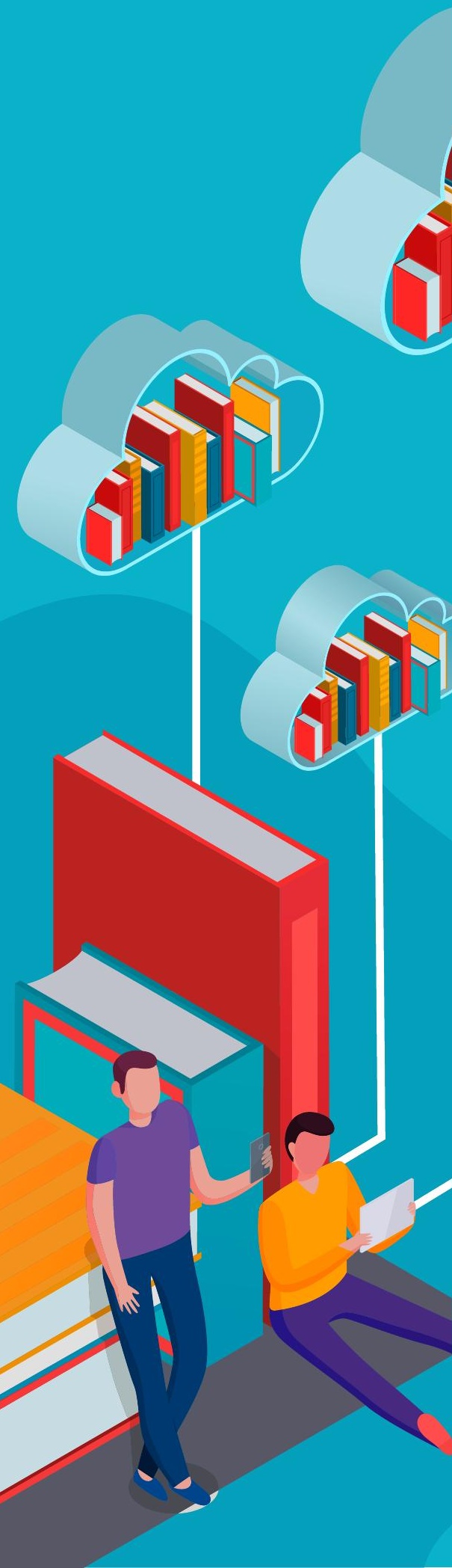




\section{La Bibliometría}

Subdisciplina de la cienciometría, proporciona a la investigación la rigurosidad necesaria; ello se lleva a cabo a través de los indicadores bibliométricos. Escorcia (2008) menciona que estos se clasifican en dos grandes grupos: indicadores de actividad e indicadores de impacto. Los primeros, visualizan el estado real de la ciencia (números, distribución, productividad, dispersión y colaboración de las publicaciones); mientras que, los indicadores de impacto, hacen referencia los Hot paper y el factor de impacto, siendo este el más reconocido.

\section{Análisis bibliométrico de la estructura del capital}

Se necesita disponer de información de la estructura del capital, de la base de datos de registros con información bibliográfica (autor, título de la contribución, de la publicación, fecha de la publicación, editorial,) almacenado y gestionado mediante sistemas informáticos. Muchas de estas bases de datos contienen descriptores, palabras claves y resúmenes y unas pocas contienen citas (índices de citas) (Ardanuy, 2012).

\section{El análisis bibliométrico en la actualidad}

La bibliometría, hasta la actualidad, evidencia tres enfoques teóricos: la primera, conocida como teoría descriptiva o clásica, debido al uso de los modelos matemáticos clásicos donde se destaca el aporte de Lotka, Bradford, Zipf, Price, Brookes, quienes se orientaron a determinar los niveles de rendimiento de los autores, además, analizar factores como frecuencia de palabras en los textos, concentración y dispersión de la información, crecimiento exponencial de la ciencia y deterioro de las publicaciones científicas (Gorbea, 2016).

El segundo enfoque teórico nace con el aporte de Narin (1976), quien confiere a esta especialidad su dimensión evaluativa y la define como "el empleo de las técnicas bibliométricas especialmente el análisis de las publicaciones y las citas, en la evaluación de la actividad científica". Este enfoque ayuda a la orientación del tema y conocimiento de 
los autores cuando se realizan investigaciones, a su vez, sirve de apoyo para ubicar al lector en el tiempo y el espacio del aporte efectuado por el autor.

El tercer enfoque aportado por Hérubel (1999), definió a la bibliometría histórica como el "estudio de los libros y revistas enmarcados en tiempo y espacio". Particularmente, los referentes temporales y espaciales ubican al investigador en la época donde sucedieron los hechos, por lo tanto, toda investigación descriptiva, histórica o evaluativa requiere de esta información.

Por lo expuesto, la bibliometría debe incluir el estudio del volumen, crecimiento, obsolescencia y difusión de literatura científica y, por otra parte, analizar la estructura social de los grupos que producen, utilizan y transmiten la ciencia para potenciar su progreso, de modo que permita formular estrategias, acciones y políticas dirigidas a orientar el curso del desarrollo de la ciencia en la comunidad académica (Amézquita, Martínez \& Maza, 2011).

\section{Utilidad de bibliometría}

La bibliometría permite conocer el alcance de las investigaciones, su pertinencia y utilidad en el campo académico y científico. Acorde a ello, Filipo (2013) sostiene que en los últimos años los estudios bibliométricos facilitan identificar grupos y áreas de excelencia, temáticas específicas, disciplinas emergentes, redes de colaboración específicas y prioridades. A pesar que esta ciencia ha sido estudiada, existen pocos avances; Juárez (2016) opina que la bibliometría puede ser reduccionista debido a que solo se limita a lo cuantitativo- numérico.

\section{Indicadores bibliométricos}

La función es determinar, medir y cuantificar cierta actividad, además de simplificar la recolección de datos. Una forma original actual del análisis bibliométrico se refiere al estudio de las referencias bibliográficas (Patrón , C.; López, J.; Piovesan, S., \& Demaría, B., 2014). 
Los índices, permiten medir:

- El crecimiento de cualquier campo de la ciencia, según la variación cronológica del número de trabajos publicados en él.

- El envejecimiento de los campos científicos, según la 'vida media' de las referencias de sus publicaciones.

- La evolución cronológica de la producción científica, según el año de publicación de los documentos.

- La productividad de los autores o instituciones, medida por el número de sus trabajos.

- La colaboración entre los científicos e instituciones, medida por el número de autores por trabajo o centros de investigación que colaboran.

- El impacto o visibilidad de las publicaciones dentro de la comunidad científica internacional, medido por el número de citas que reciben por parte de trabajos posteriores.

- El análisis y evolución de las fuentes difusoras de los trabajos, por medio de indicadores de impacto de las fuentes.

- La dispersión de las publicaciones científicas entre las diversas fuentes, etc. González (1997).

Por su parte, Filippo (2013) sostiene que los indicadores en la bibliometría aportan a la difusión y transmisión del conocimiento generado en la investigación. Son válidos cuando los resultados de la investigación se transmiten a través de publicaciones científicas y técnicas. Además, para evaluar la actividad científica de manera correcta, se han de utilizar varios indicadores y no exclusivamente uno, ya que la información proporcionada sería sesgada y nos daría una idea falsa de esta actividad. 


\section{Clasificación de indicadores bibliométricos}

Estos indicadores bibliométricos constituyen una herramienta adecuada para examinar las actividades científicas, siempre que sean utilizadas adecuadamente. Villaroel (2014) expone que no todos los indicadores se pueden utilizar en los diferentes documentos, debido a la falta de información en algunos.

Tabla 1. Clasificación de indicadores bibliométricos

\begin{tabular}{|c|c|}
\hline INDICADORES & CARACTERÍSTICAS \\
\hline \multirow{8}{*}{ Indicadores personales } & Edad de los investigadores \\
\hline & Sexo de los investigadores \\
\hline & Antecedentes personales \\
\hline & Índice de productividad personal \\
\hline & Índice de colaboración \\
\hline & Índice de multi autoría \\
\hline & Índice institucional \\
\hline & Índice de transitoriedad \\
\hline \multirow{3}{*}{ Indicadores de citación } & Índice de antigüedad obsolescencia \\
\hline & Factor de impacto de las revistas \\
\hline & Índice de auto citación \\
\hline \multirow[t]{3}{*}{ Indicadores de contenido } & Temáticos o textuales \\
\hline & Descriptores \\
\hline & Paradigma adoptado \\
\hline \multirow{4}{*}{$\begin{array}{l}\text { Indicadores } \\
\text { metodológicos }\end{array}$} & Teoría desde o para lo que se trabaja \\
\hline & Diseños específicos utilizados \\
\hline & Riesgos muestrales \\
\hline & Técnicas de análisis \\
\hline
\end{tabular}

Fuente: Estudio Longitudinal de la Producción Española (Vallejos, 2005). Elaborado por la autora. 
Velasco \& Eiros (2012) indican que este tipo de indicadores brindan una valiosa información sobre la situación de la investigación y complementan al juicio de expertos; su finalidad depende, en gran medida, del uso adecuado que debe hacerse con conocimiento de sus limitaciones.

Rueda \& Villaroel (2005) sostienen que para el análisis bibliométrico es necesario el uso de los siguientes indicadores:

- Cuenta de artículos: se calcula al contabilizar el número de artículos incluidos en una publicación durante un año particular (solamente investigación original y reseñas). Permite al lector conocer el volumen de publicaciones emitidas durante un periodo.

- Factor de impacto: se aplica a las revistas y permite evaluar y comparar la importancia de la misma frente a otras de su mismo campo. Este se calcula como el cociente entre el número de citas a artículos de dicha revista y el número total de artículos publicados, tomando solo la suma de los valores de los dos últimos años.

Escorcia, T. (2008) menciona ciertas limitaciones de este tipo de indicadores:

- En el uso de las citas, por ejemplo, no existe un modelo aceptado que explique el proceso de citación; por ello, se recomienda tener buenos argumentos $y$, sobre todo, utilizar publicaciones con prestigio internacional con la finalidad de incrementar la credibilidad y pertinencia de las publicaciones presentadas.

- Problemas técnicos. Al usar las bases de datos aparecen errores tipográficos, por ejemplo, en el nombre del autor, como la referencia bibliográfica, la existencia de homónimos, el distinto tipo de firmas de los autores en el transcurso del tiempo. 
- La rapidez en el que la bibliografía se vuelve obsoleta en áreas científicas; esto es difícil de comprar.

Morocho (2018) señala la utilidad de los indicadores que permiten:

- Caracterizar y evaluar unidades de análisis por métodos cuantitativos, basados en medidas genéricas o en la cuantificación de opiniones de expertos. Los requisitos básicos son: robustez, validez de medición y aplicación, así como reproducibilidad.

- Los indicadores deberían ser insensibles a los cambios marginales en los aspectos que pretenden medir; deben ser medidas significativas de lo que son aplicadas en las mismas condiciones y utilizando los mismos datos y métodos, idénticos valores de indicador que deben ser obtenidos.

- Un indicador debe ser significativo, pero, formalmente, no bien definido o a la inversa; puede ser matemáticamente correcto, pero, no una medida significativa. 


\section{Principales bases de datos académicos}

Las bases de datos académicos constituyen los

primeros canales de comunicación y transmisión de investigaciones dado al prestigio en el manejo de las revistas ya que brindan confianza a los investigadores y usuarios en general. A continuación, se describe a las revistas de mayor prestigio.

- Scopus: es una base de datos bibliográfica de resúmenes y citas de artículos de revistas científicas. Cubre aproximadamente 18 mil títulos de más de 5 mil editores internacionales, incluyendo la cobertura de 16.500 revistas revisadas por pares de las áreas de ciencias, tecnología, medicina y ciencias sociales, incluyendo artes y humanidades. Scopus, también proporciona herramientas bibliométricas que miden el rendimiento de publicaciones y autores, basadas en el recuento de citas recibidas por cada artículo (Universidad Católica de Chile, 2018).
- Latindex: es un sistema de información sobre las revistas de investigación científica, técnico-profesionales y de divulgación científica y cultural que se editan en los países de América Latina, el Caribe, España y Portugal. La misión del sistema es difundir, hacer accesible y elevar la calidad las revistas académicas editadas en la región, a través del trabajo compartido; ofrece datos bibliográficos y de contacto de todas las revistas registradas, ya sea que se publiquen en soporte impreso o electrónico (Escuela Politécnica Nacional, 2017).

\section{Scopus}




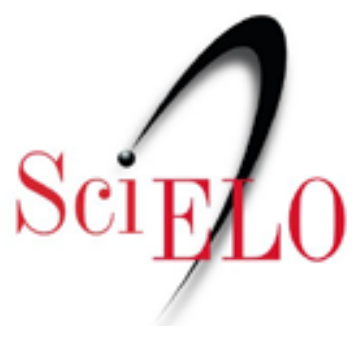

- SciElO: este programa permite la publicación electrónica de ediciones completas de revistas científicas, la organización de bases de datos, la recuperación de textos por su contenido y la producción de indicadores estadísticos de uso e impacto de la literatura científica. El programa opera regularmente desde junio de 1998 permitiendo difundir rápidamente la información científica generada localmente (Fillipo, 2002).

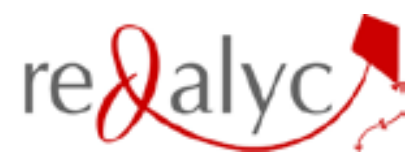

- Redalyc: (Red de Revistas Científicas de América Latina, el Caribe, España y Portugal) forma parte de un conjunto de hemerotecas científicas en línea de libre acceso; ha evolucionado con el transcurso del tiempo a un sistema de información científica que incorpora el desarrollo de herramientas para el análisis de la producción, la difusión y el consumo de literatura científica (ECURED, 2018).

Para el análisis de la estructura de capital se utilizará la base de datos bibliográficos Scopus, debido a que ofrece un mejor servicio de indización de temas de interés a nivel mundial y es la fuente de consulta preferida por los usuarios, porque resume gran cantidad de artículos (Salazar y Bautista, 2017); además, indiza revistas de edición científica de las cuales el $71.4 \%$ son de acceso por paga y el $26.3 \%$ de acceso libre, a diferencia de otras bases de datos como Redalyc y Scielo que se caracterizan por publicar e indexar tanto en revistas como en artículos de repositorios digitales (Miguel, 2011); mientras que, Latindex es la base de información más completa de revistas académicas expuestas en un catálogo (Alperin, \& Babini, 2014). Así mismo, en los rankings de Scopus según SClmago (2018), los indicadores de producción son del $8 \%$, de impacto $13 \%$, publicaciones de alta calidad $2 \%$, excelencia $2 \%$ que corresponden a los valores máximos de calificación. 


\section{METODOLOGÍA}

\section{Búsqueda bibliográfica}

La manera más usual de buscar referencias bibliográficas de resúmenes y citas de artículos de revistas científicas, es mediante la ubicación del tema concreto o nombre de su autor. Para el presente caso, hemos utilizado los siguientes descriptores en inglés: capital estructure.

\section{Ingresar a Scopus para conseguir bibliografía científica}

Se ingresó a la base de datos bibliográficos de resúmenes y citas de Scopus, se adoptó al descriptor, la expresión estructura de capital y, en el banco de datos, se hizo una búsqueda de los textos de interés. Luego se procedió a determinar los documentos en relación al estudio que se está haciendo: estructura de capital, valoración, riesgo, endeudamiento. Bajo estos parámetros, se seleccionaron los indicadores bibliométricos de contenido que ayudaron a identificar las investigaciones concernientes al tema.

Con estos antecedentes, se seleccionó la fuente de datos, esto hace referencia a las revistas y artículos nacionales para conocer la participación de aporte académico. Una vez seleccionada la fuente, se prosiguió con una o varias bases de datos iniciales y se depuró para corregir errores de escritura, considerando las necesidades de la investigación.

\section{Criterios de selección}

El primer momento de esta investigación fue la revisión bibliográfica, enfocada en la bibliometría y en los documentos escritos por investigadores en el área de las Finanzas y Contabilidad. Las unidades de análisis fueron: artículos, revistas y resúmenes. 


\section{Las variables a estudiar fueron:}

- Número de Publicaciones: Este indicador representa la medida básica del volumen de salida; está enlazada directamente a las características de la comunidad investigadora. En este trabajo se consideró todos los documentos de análisis de la estructura del Capital.

- $\quad \mathrm{PC}=$ Número de documentos publicados en SCOPUS cuyo tema es "Estructura del Capital".

- Número Total de citas: Este indicador de impacto académico inicia en la temática y tipo de documento que permite conocer el impacto de los trabajos científicos en el entorno científico.

- Colaboración: Este indicador tiene que ver con el tipo de autoría de los trabajos; estos pueden ser individuales o grupales. Para Alvarado (2011), la colaboración es un elemento clave en el progreso del conocimiento y en la productividad de la investigación en cualquier campo y se habría iniciado como respuesta a la profesionalización de las disciplinas y al crecimiento de los conocimientos.

- Universo de estudio: Estuvo conformado por todos los artículos, revistas e investigaciones de carácter científico, tomando en cuenta los últimos 5 años; es decir, de 2013 a 2018 y que contengan como tema de estudio Estructura del Capital.

Cabe indicar que este universo de estudio, estuvo sometido a ciertas cuantificaciones para la selección como, por ejemplo, en la clasificación de revistas y la selección de estudios en un periodo de 5 años antes, el factor de impacto (número de citas) esto para evitar la obsolescencia científica que, según Ebrain (2016), puede ocurrir por diversas razones: su información es válida, pero, ha sido incorporada en una publicación posterior (obliteración), ha sido superada por algún último trabajo, el área de investigación ha perdido importancia o, simplemente, la información ha dejado de tener valor. 


\section{Recuperación de la información}

Los datos utilizados fueron obtenidos de Scopus Elseiver, debido a que posee mayor base de información que contienen referencias bibliográficas facilitando tener una visión multidisciplinaria de la actividad científica e innovación tecnológica, las cuales, cuentan con una opción de descarga. Considerando estos parámetros se obtuvo los datos de la base de datos Scopus, mediante el ingreso a través del enlace //www. scopus.com/home.uri.

Figura 1. Ingreso a Scopus

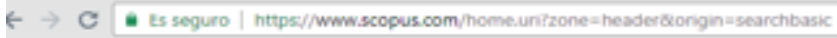

Scopus Preview

Author search

Sources

Help $\checkmark$

\section{Welcome to Scopus Preview}

What is Scopus $x \quad B \log$ 入 in

\section{Scopus content}

$>$ Content coverage guide

$>$ Scopus source list

$>$ Book title list

\section{Check out your free author} profile!

Did you know Scopus offers free profiles to all

indexed authors? Review yours, claim it. and

update it - all for free!

Fuente: Scopus (www.scopus.com)

Elaborado por la autora.

Posteriormente, se ingresó descriptores en inglés: capital estructure en la opción Search en la pestaña con el nombre Sources. Subject área (Economics, econometrics and Finance y Business, Management and Accounting). 
Figura 2. Búsqueda Estructura del Capital

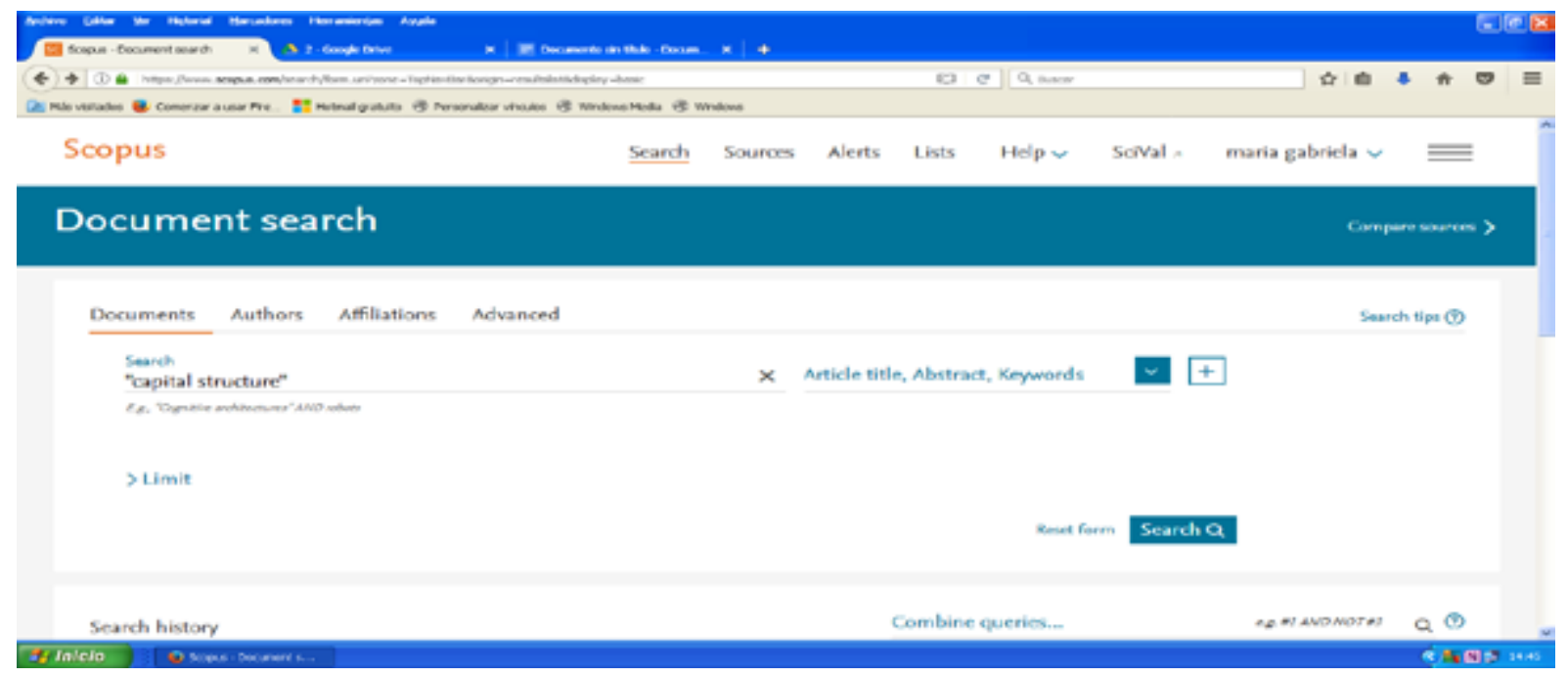

Fuente: Scopus (www.scopus.com)

Elaborado por la autora.

Obtenidos los resultados, se procedió a la descarga de los documentos relacionados al tema principal de estudio sobre estructura de capital, la que se hizo por los últimos cinco años y considerando información a nivel mundial en idioma inglés para determinar el aporte científico que ha producido. 
Figura 3. Extracción de resultados de documentos

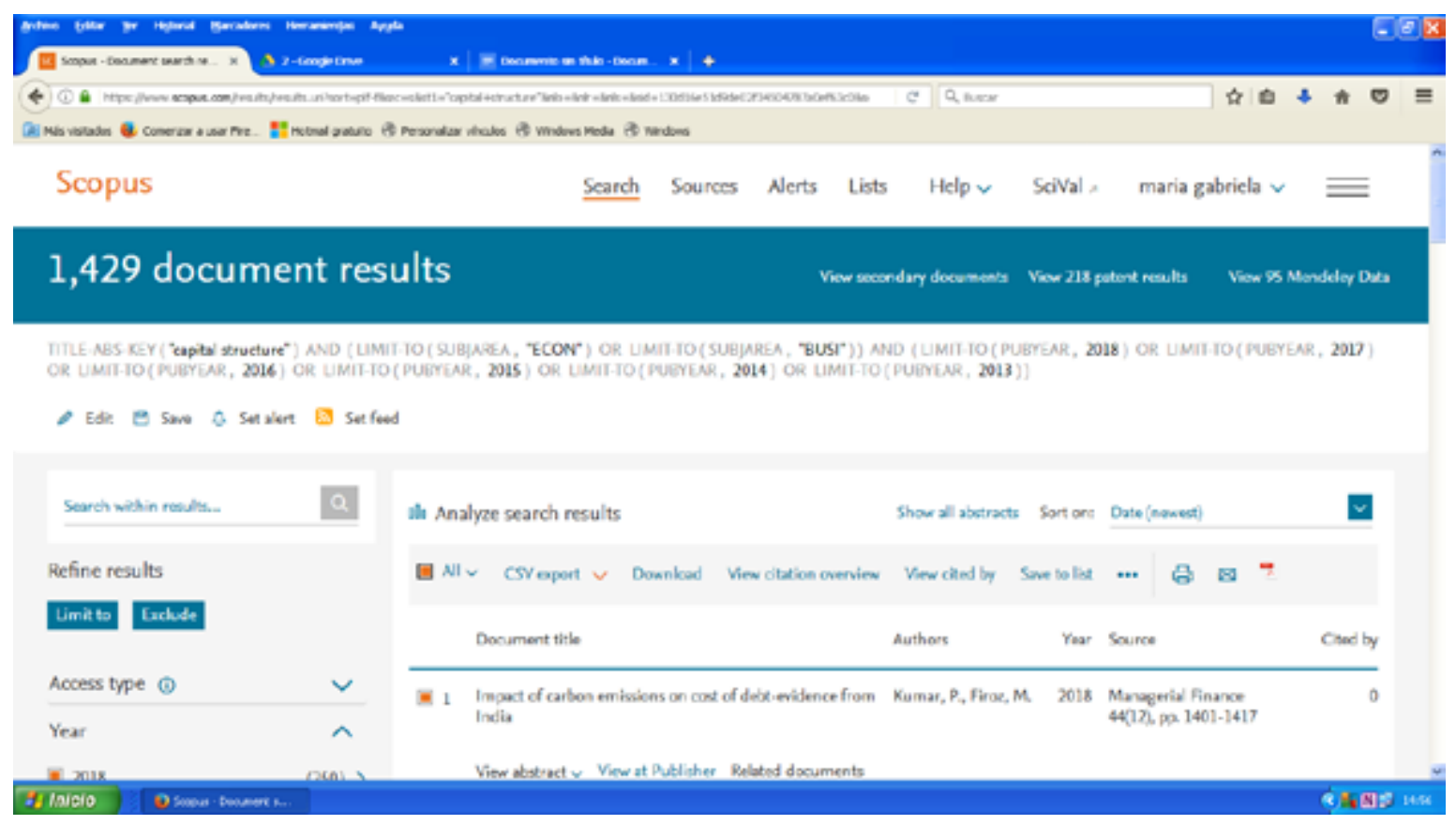

Fuente: Scopus (www.scopus.com)

Elaborado por la autora.

A través de la opción Export, se seleccionaron los datos a ser descargados según Subject área (Economics, econometrics and Finance y Business, Management and Accounting) mediante el formato bid; se recolectó los datos asociados a Source List y Bibliographical información.

Para el análisis cualitativo se procesó la información en el programa ATLAS.ti. V8; finalmente, se obtuvo las conclusiones cualitativas y cuantitativas en relación al aporte mundial de publicaciones capital estructure. 


\section{Evaluación de la calidad de los artículos seleccionados}

La calidad de los artículos seleccionados es fundamental en el transcurso de producción y elección de la literatura científica del análisis de la estructura del capital, mediante la identificación de las escalas de evaluación de la calidad metodológica de los artículos, de acuerdo a la cantidad de items, aplicaciones y limitaciones.

Quillis (2015), menciona que al contar con publicaciones se asume implícitamente que todas portan la misma cantidad de conocimiento científico. El prestigio de las fuentes bibliográficas donde se publica el trabajo, representa una medida de la influencia que este puede ejercer.

Cabe indicar, también, que para constatar la validez de la información se consideró ciertos indicadores de calidad, los cuales, según Martín (2018), son:

- Calidad del contenido de la investigación, identificación de los miembros de los comités editoriales y científicos, identificación de los autores (nombre, apellidos, afiliación), contenido de la revista, instrucciones detalladas a los autores. Artículos acompañados de un resumen; si puede, ser resumen en al menos dos idiomas e incluir también palabras clave.

- La editorial o el comité de selección debe incluir las razones para la aceptación, revisión o rechazo del manuscrito, así como los dictámenes emitidos por los expertos externos.

- Periodicidad de las revistas y regularidad y homogeneidad de la línea editorial en caso de editoriales de libros. Que conste claramente la entidad editora.

- Anonimato en la revisión de los manuscritos.

- Cantidad de citas que recibe el artículo por parte de otros usuarios, este se denomina factor de impacto. Se usa comúnmente para determinar la visibilidad de un autor, de una disciplina o de una revista. 


\section{RESULTADOS}

\section{Análisis de la variabilidad, fiabilidad y validez de los artículos}

Para determinar la variabilidad, de los artículos de estructura de capital, mediante la utilización de indicadores, se obtuvo los siguientes valores:

Figura 4. Número de documentos publicados por año

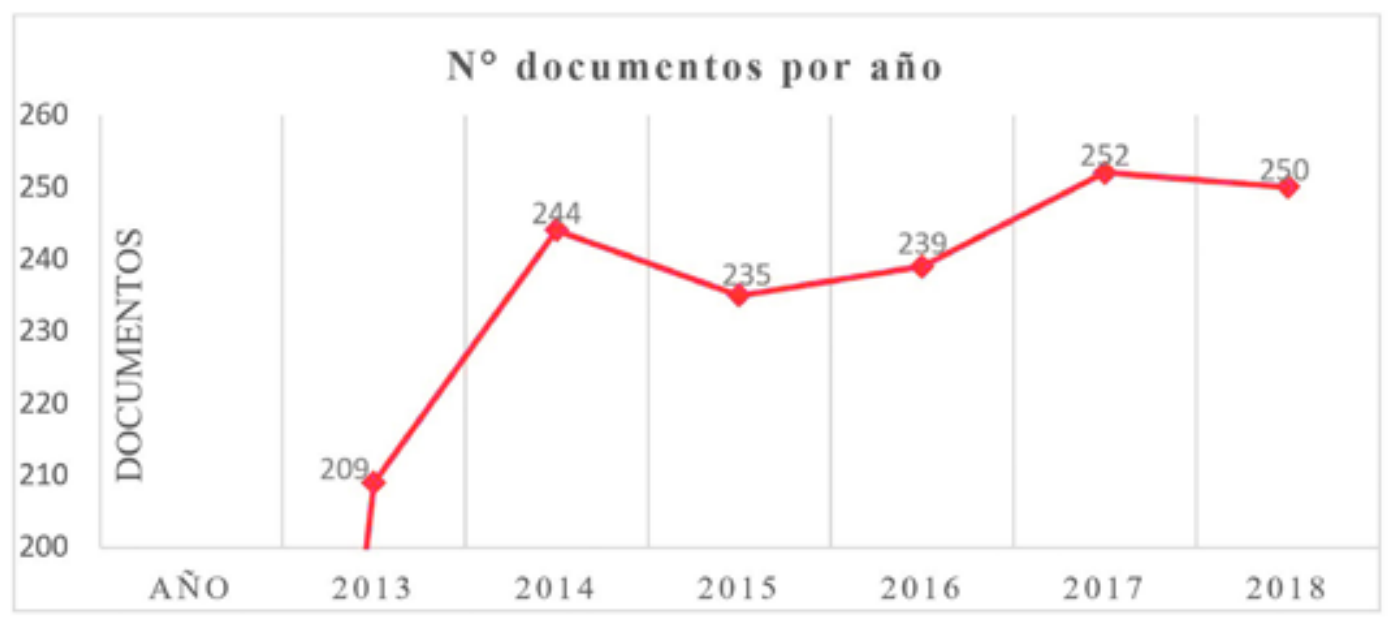

Fuente: Scopus (www.scopus.com)

Elaborado por: Gabriela González. 
A base del análisis bibliométrico sobre la producción científica en las revistas más relevantes presentes en la base de datos Scopus, sobre Capital Estructure durante el periodo 2013-2018, se determinó que el número de artículos publicados sobre la temática propuesta muestran una curva irregular; la producción del 2013 al 2014 se incrementa considerablemente a pesar que al año 2015 decae, sin embargo, del 2016 al año 2018 muestra un leve progreso de la actividad científica, el total de temas del periodo mencionado es de 1429 publicaciones.

Figura 5. Tasa de colaboración neta

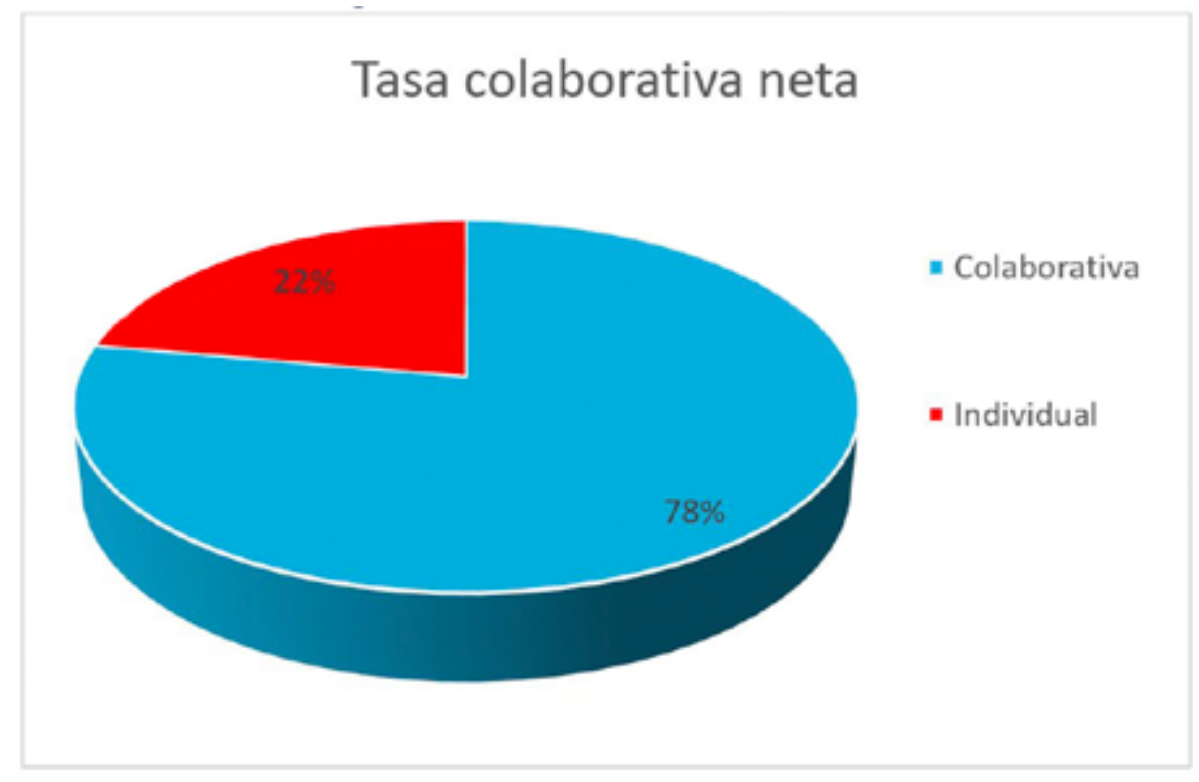

Fuente: Scopus (www.scopus.com)

Elaborado por: Gabriela González.

En la Figura 5, se puede observar la tasa de colaboración neta, la cual, se estructura de los trabajos realizados de forma colaborativa y de forma individual. Se encontró que el $78 \%$ de investigaciones son realizadas de forma colaborativa y el $22 \%$, de forma individual. 


\section{Contextualización de las principales teorías de la estructura del capital}

El estdio teórico y experimental sobre la estructura de capital, ha tenido un significante progreso a partir de los años 50 hasta mediados de los años 70; se direccionó hacia la modernización, siendo el punto central de análisis, el comportamiento de la cartera, con el aporte de investigadores como Markowitz (1952), y la teoría financiera Modigliani \& Miller (1958), base que dio inicio al estudio de la estructura de capital.

\section{Teoría de jerarquía de preferencias Pecking Order}

Myers (1984), expone que las organizaciones eligen financiarse con capital propio mediante la reinversión de las utilidades para beneficiarse de las circunstancias favorables de inversión que se presentan en el mercado.

Esta teoría surge para responder la incógnita del porqué grandes y sofisticadas empresas con una rentabilidad muy alta poseen bajos niveles de endeudamiento. Esta teoría se basa en la suposición de que existe información asimétrica entre la gerencia de la empresa y los inversionistas (Orozco \& Quijano, 2014).

La teoría de jerarquía Pecking Order, actualmente se caracteriza por poseer una mayor aceptación entre las empresas, muchas de ellas, a criterio de Moreira \& Rodríguez (2006), están fundamentadas en la carencia de una estructura óptima de capital, por lo que trabajan para fomentar sus nuevos proyectos con recursos propios para prevenir circunstancias desfavorables en el mercado como consecuencia de la poca o mala información que no brinda confiabilidad para la ejecución de las inversiones. Zambrano \& Acuña (2013) fundamentan que Pecking Order, en la actualidad, tiene gran importancia para las empresas por el propósito de financiar sus nuevas inversiones con recursos propios.

Mejía (2013) alega que existen tres fuentes de financiamiento opcionales a ser utilizadas, la principal son las utilidades retenidas como recursos propios; en caso de no ser suficientes, las empresas acuden al financiamiento exterior mediante deuda financiera considerando siem- 
pre el menor costo. Cuando este recurso es limitado o se agota, como segunda alternativa es la emisión de bonos y, como tercer recurso extremo, la emisión de acciones.

Bajo esta argumentación, se puede entender que la teoría de jerarquía de preferencias no brinda una combinación adecuada que equilibre los beneficios y desventajas de la deuda, más bien constituye la planificación de estrategias por parte de los gerentes o administradores de la organización para acrecentar sus inversiones, manejando una jerarquía de particularidades en cuanto a la utilización de las fuentes de financiación.

\section{Teoría del Óptimo financiero Trade Off}

Fernández (2017) afirma que esta teoría, denominada teoría estática fue propuesta por Kraus \& Litzenberger (1973), bajo la hipótesis del equilibrio entre los costos de quiebra y las ventajas que generan beneficio de ahorro de impuestos por la deuda.

Esta conjetura teórica se simplifica en el hecho de la presencia de la combinación adecuada entre la deuda y el capital, agregando valor económico a la organización y se obtiene luego que los beneficios y costes procedentes de la deuda se nivelen (Shyam y Myers, 1994). Sin embargo, Coronel (2015) respalda el criterio de Kim (1978), quien contrapone argumentando que un elevado nivel de deuda en la organización podría ocasionar un amenazador riesgo de impago por parte de la empresa, lo que constituye una amenaza tanto interna como externa. Al decir interna, se refiere a las dificultades para mitigar el problema y externa, por el hecho del deterioro de la imagen de la organización frente a las competencias.

Revelo, Rodríguez \& Mantilla (2017) mencionan que la Teoría del Trade Off favorece para que los administradores de las organizaciones busquen la estructura financiera óptima y mantengan como objetivo un nivel de apalancamiento limitado, para suplir las ventajas fiscales y la posibilidad de quiebra cuando este coeficiente se incremente. A su vez Escobedo (2016) asegura que un método para mejorar la estructura financiera es disminuir el costo de las fuentes que intervienen en el 
financiamiento de los activos de la empresa. Contreras \& Diaz (2015) señalan como ejemplo para mejorar la estructura óptima, descontar los flujos de caja esperados al costo promedio ponderado de financiamiento; es decir, entre menos costosos sean los recursos financiados, el valor de la organización será mayor. Bajo este criterio, rubros como costos, minimizan las ventajas a elementos como la deducibilidad fiscal de las cuentas de gastos de deuda, beneficiando a la obtención de índices óptimos de endeudamiento.

Retomando la perspectiva de beneficios de la teoría de Trade off, Hackbarth, Hennessy y Leland (2011) argumentan que la estructura de la deuda óptima para las organizaciones débiles implica financiamiento directo con entidades bancarias, mientras que las empresas que se encuentran en un nivel óptimo tienen la opción de utilizar la deuda financiera y de mercado.

¿Será acaso que los factores económicos, políticos, entre otros, influyen directamente en las actividades empresariales?, sin duda alguna sí; estas variables inciden en el movimiento económico de las organizaciones, en la cantidad de adquisición de bienes o servicios que generan las utilidades a las empresas.

\section{Teoría de la señalización Signaling}

Vargas (2014) resume la teoría de la señalización desde la estructura de capital fundamentada en las complicaciones de información asimétrica entre gestores e inversores propuesta por Ross (1977); además expresa que Signaling fue estudiada desde otras perspectivas como, por ejemplo, mercados de productos analizada por Akerlof (1970) del mercado laboral, propuesta por Arrow (1971) y Spence (1973).

Enfocando la teoría de la señalización desde la estructura de capital es examinada desde las complicaciones que se presentan entre administradores o representantes de una organización y los inversionistas, de acuerdo al manejo de los directivos en relación a la información interna, que puede ser transferida en cualquier momento a los inversionistas externos, dando lugar a la interferencia en que los precios de 
las acciones se eleven; sin embargo, la información no siempre es confiable y cierta como para asumir una acción, en este caso, de inversión.

En relación a Signaling, la emisión de deuda actúa como una señal efectiva que incide en el valor de la organización; mientras que, la emisión de acciones es contraproducente e indica una sobrevaloración de la estructura empresarial. (Fernández, 2017).

Vargas (2014) aporta a esta problemática, bajo el criterio de Ross (1977), indicando que se puede dar solución mediante la adopción de una política financiera a base de indicaciones mediante señales entendibles para los accionistas, la que sirve para la toma de decisiones; por otra parte, sugieren que esta medida posee cierta restricción como, por ejemplo, un coste sumamente elevado.

Dittmar (2001) contrapone el criterio antes mencionado aduciendo que las señales del coste cero y señales costosas no son adecuadas para que los administradores comuniquen a los inversionistas que existen excelentes expectaciones en relación al desempeño de la organización, ya que esta actitud puede ser utilizada por cualquier empresa; $\sin$ embargo, si se considerara el aumento del apalancamiento financiero, marcaría la diferencia, ya que toda organización no se encuentra en posibilidad de llevar a cabo un anuncio de tal magnitud.

Cabrera \& Rico (2015), al igual que Escobedo (2013) y Veronesi (2000), opinan que bajo esta estrategia de señalización optada por los gerentes las empresas deben incrementar su endeudamiento, a diferencia de aquellas organizaciones cuyo riesgo, a causa de esta señalización, les llevaría a ubicarse en una posición de sobre valoración y cargas como adición de préstamos se convierten en un riesgo, que podría llevarles a la quiebra. 


\section{La estructura de capital desde el análisis Bibliométrico}

En la actualidad, la estructura del capital ha sido de gran utilidad para la evaluación de las empresas en dimensiones como: procesos de incorporación, negociación, inversión, entre otros. En este contexto, estas operaciones se convierten en estrategias competitivas que son utilizadas por las empresas para enfrentar al mercado cambiante, por ello, surge la necesidad de determinar el valor real de una empresa.

La revisión de literatura sobre Análisis Bibliométrico de la Estructura del Capital, permite conocer las investigaciones efectuadas por autores. A continuación, citaremos las siguientes:

Bernal (2013) llevó a cabo un estudio bibliométrico en Scopus Preview sobre finanzas durante el periodo 2004-2013. El objetivo general de este trabajo de investigación fue diseñar una red de autores y coautores de artículos científicos en finanzas de 2004 a 2011 de Scopus Preview. Los tipos de documentos fueron 4931 artículos y 769 revisiones; los títulos de la fuente más representativos: European Journal of Operational Research; Quantitative Finance; Journal of Banking and Finance; Management Science; Construction Management and Economics; Journal of Financial Economics; Economics of Education Review; Journal of the Operational Research; Society; Operations Research y Strategic Management Journal.

A pesar de la importancia del estudio de la estructura del capital, los resultados obtenidos durante siete años de referencia representan poca información. A continuación, se observan en la siguiente figura: 
Figura 6. Estudio bibliométrico: Área Finanzas y Ciencias Sociales

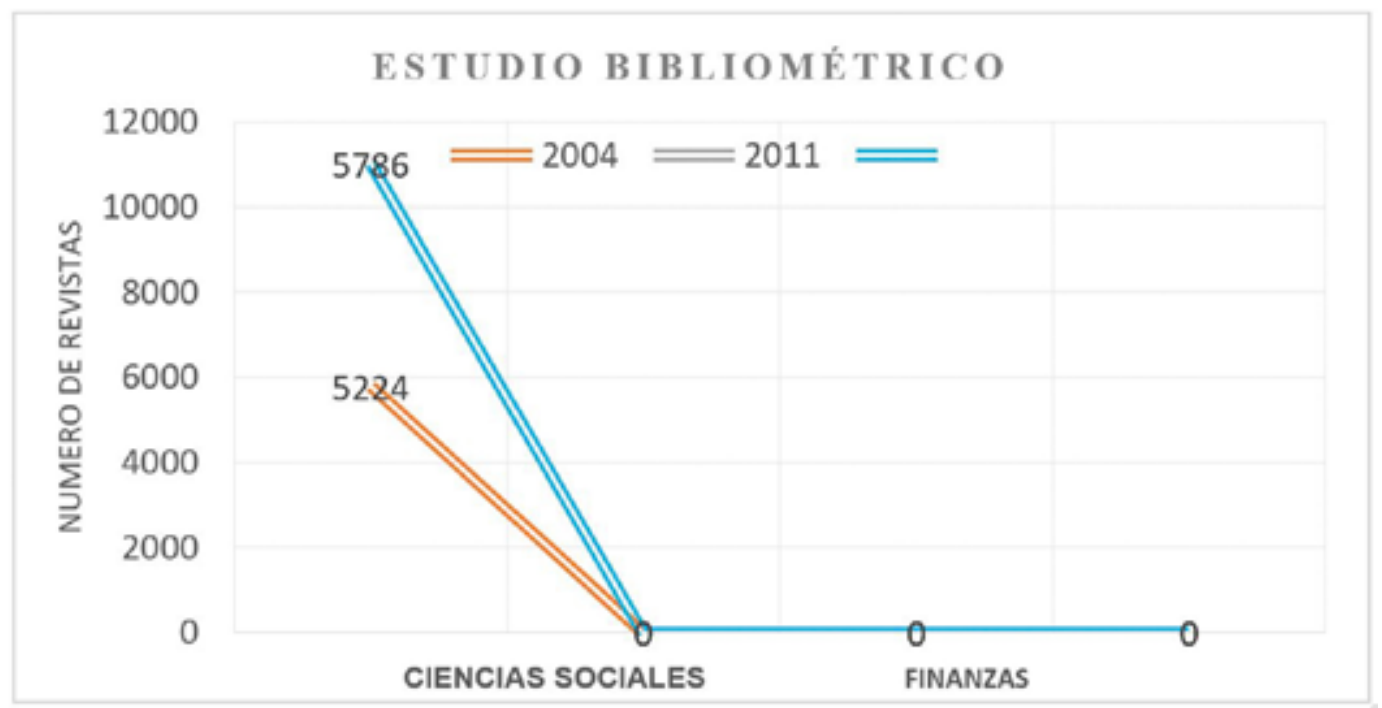

Fuente: Un estudio bibliométrico en Scopus sobre finanzas (Bernal, 2013). Elaborado por: Gabriela González.

Se llegó a concluir que el análisis bibliométrico efectuado por Bernal (2013), permitió identificar que de 2004 a 2011 en la base de datos Scopus Preview se contaba con 5786 documentos con el tema de finanzas, de los cuales, el $86.51 \%$ fueron artículos científicos y que el 92.42\% son escritos en el idioma inglés; los países que publicaron más fueron Estados Unidos de América y Reino Unido.

Existen otros estudios relacionados a la estructura financiera como Importancia de la investigación científica en ciencias financieras y contables, efectuado por Correa (2013), cuyo objetivo fue analizar la actividad dentro del ámbito de la economía financiera; para ello, se utilizaron las palabras clave como estructura de capital, mercados financieros, además del registro del volumen de información.

Otro tema fue el desarrollado por Nobre \& Rueda (2016) que llevaron a cabo un estudio denominado Estructura del capital: Relevamiento de la literatura y desarrollo reciente en el área. Para la investigación de este tema recopilaron la información a nivel mundial y por países, a fin de determinar el aporte desde el ámbito de la administración, economía y ciencias sociales. 


\section{DISCUSIÓN}

\section{Organización y estructuración de los datos}

Se presenta la información del análisis Bibliométrico a base de los resultados cuantitativos adquiridos con la información de Scopus y los resultados cualitativos a través de programa ATLAS.ti. V8.

\section{Análisis cuantitativo}

Se evidenciaron 1.429 documentos relacionados a capital estructure según Subject área: Economics, econometrics and Finance y Business, Management and Accounting, durante el periodo 2013 - 2018.

Figura 7.Documentos por área temática

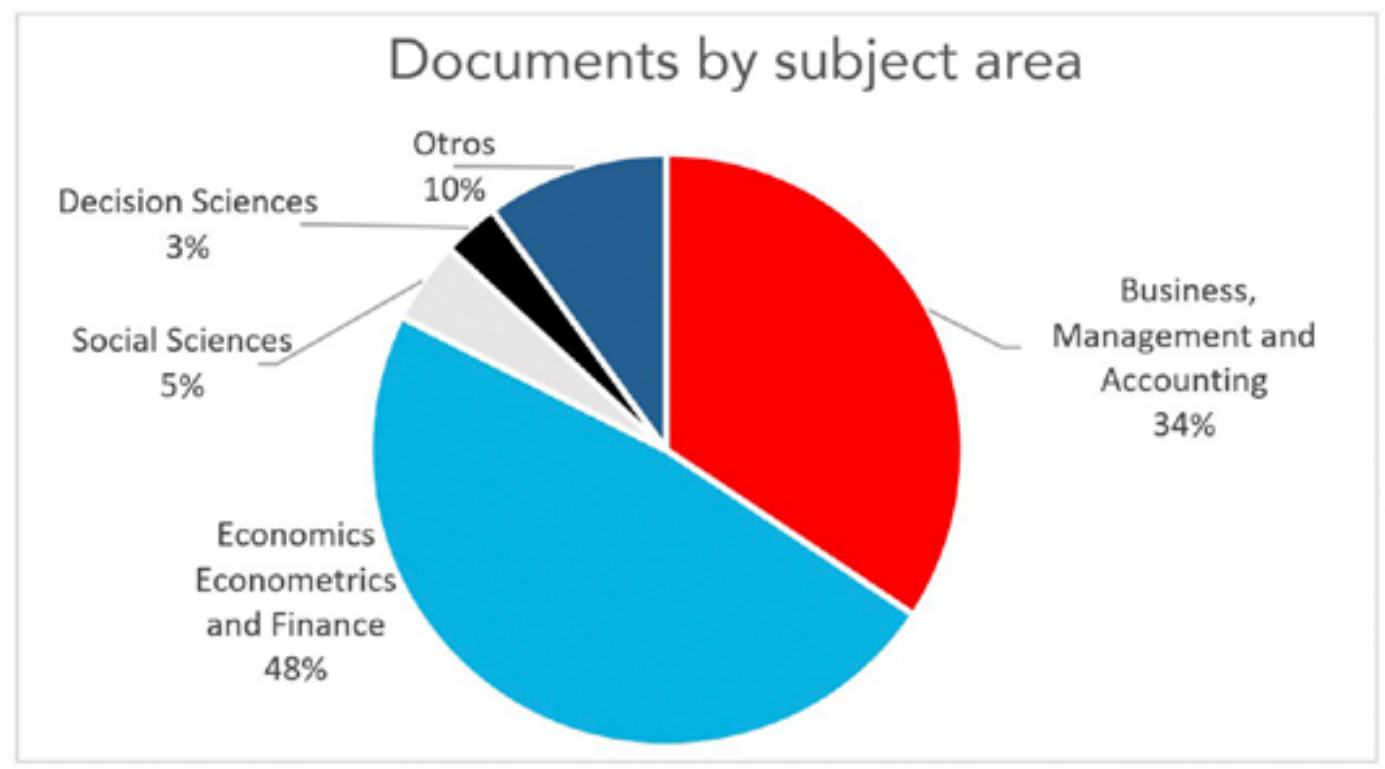

Fuente: Scopus (www.scopus.com) Elaborado por: Gabriela González. 
Economics Econometrics and Finance registra un total de 687 publicaciones que representan el 48\%; Business, Management and Accounting posee el 34,40\% que equivale a 491 publicaciones; entre estas dos áreas se concentra el mayor número de literaturas con un total del $82,4 \%$. En menores cantidades se observa en las áreas de Social Sciences con 64 documentos que equivale 4,5\% y Decisión Sciences tiene 44 títulos con el 3.10\%; otras áreas como Mathematics, Engineering, Environmental, entre otras, representan el $10 \%$, similar a 143 publicaciones.

Figura 8. Bibliométricas relacionadas a estructura de capital por tipo

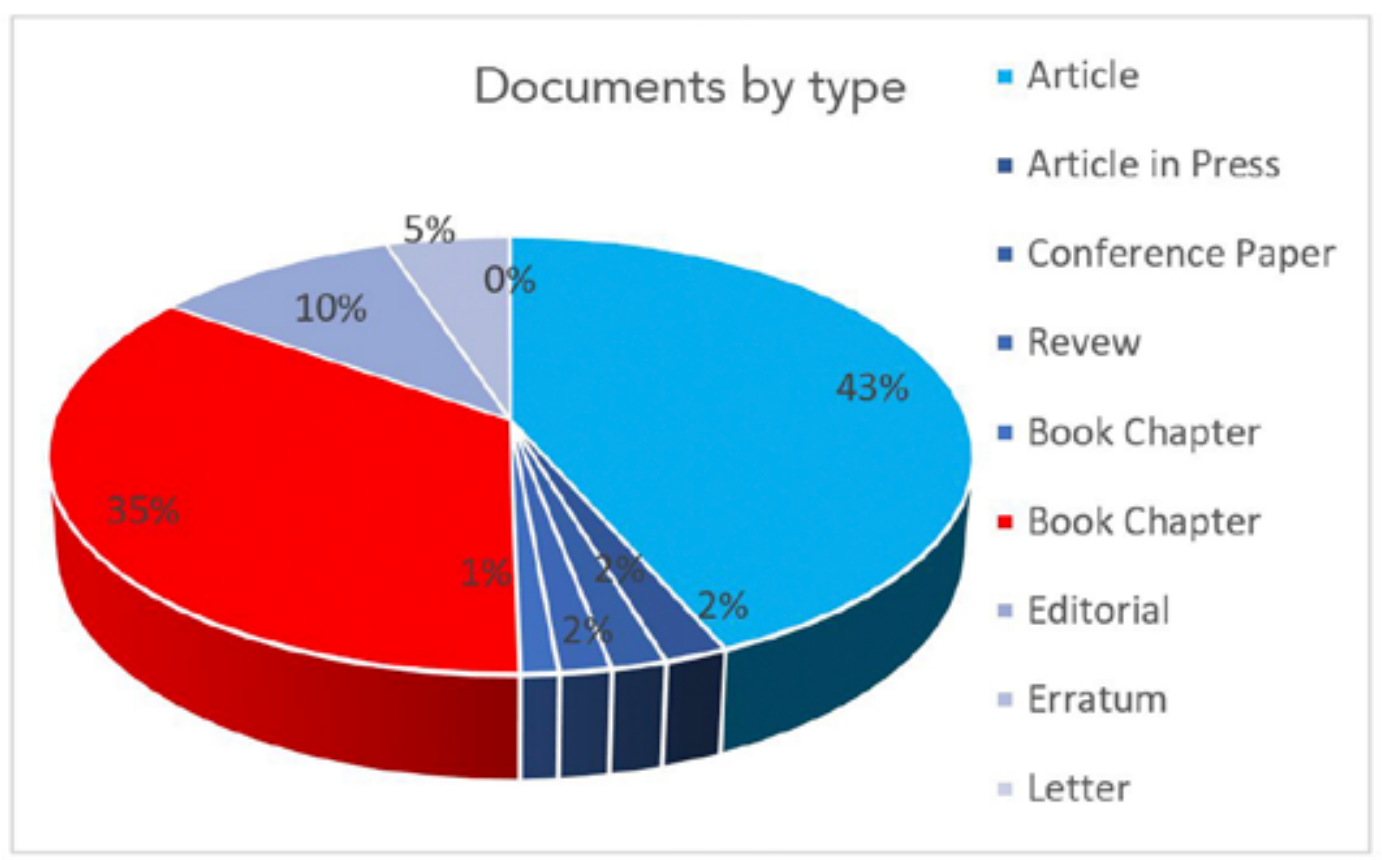

Fuente: Scopus (www.scopus.com) Elaborado por: Gabriela González. 
Las bibliométricas, en relación a la información correspondiente al tipo de documentos investigados, se conoció que, de las 1.429 fuentes bibliográficas, 1.237 son artículos que se caracterizan por ser investigaciones publicadas en revistas especializadas como aquellas que se mencionó anteriormente Redalyc, Scielo; publicaciones en prensa se encontraron 53 temas, libros 10, capítulos en libros 33, documentos de sesión 47 y en menor número otras revisiones.

Figura 9. Documentos según autor

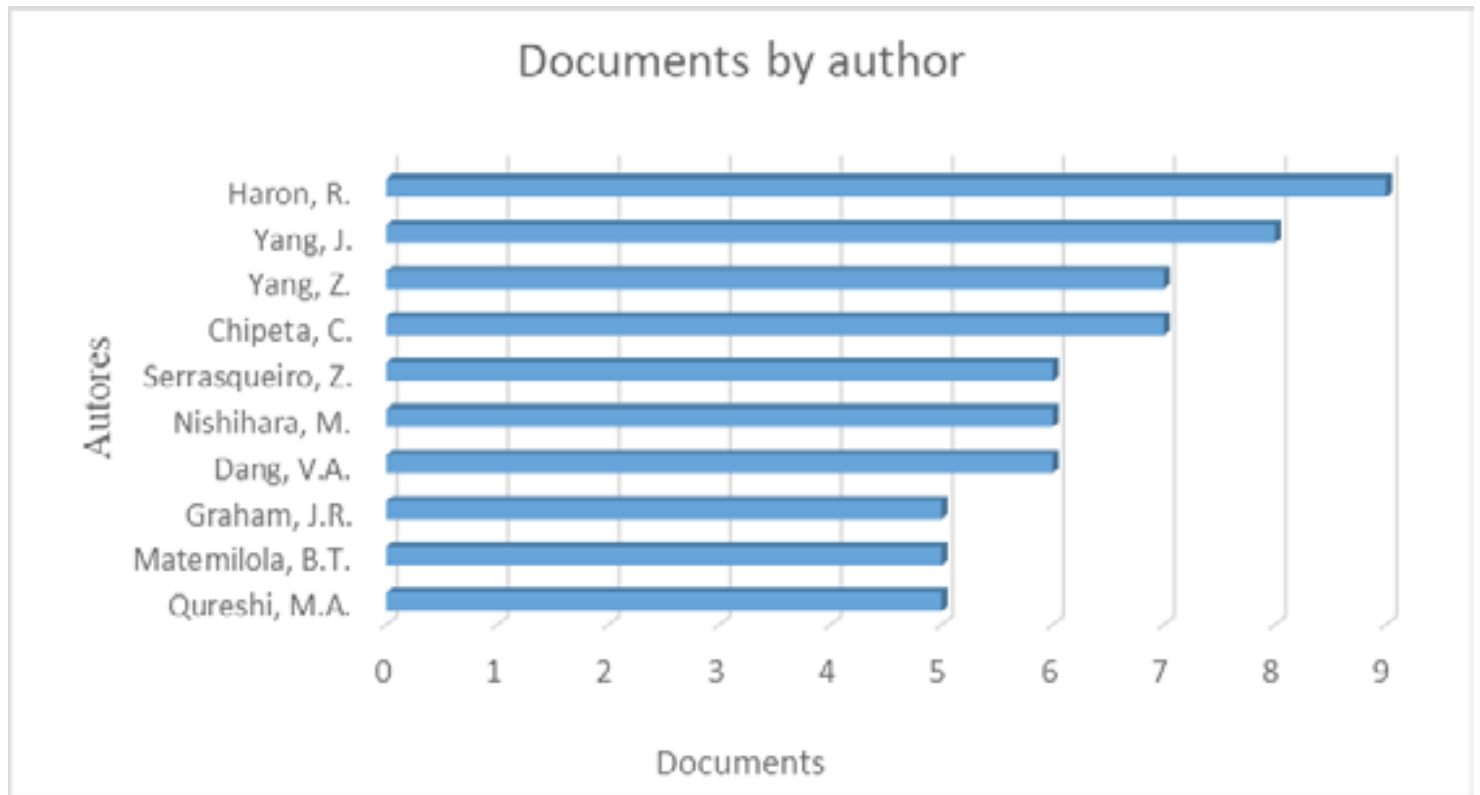

Fuente: Scopus (www.scopus.com)

Elaborado por: Gabriela González.

Como se aprecia en la figura 9, existen autores como Haron, Yang, J., Yang, Z., Chipeta, Serrasqueiro, Nishihara, Dang, entre otros, que han aportado con varios documentos de investigación. A continuación, se presenta los autores más citados durante el desarrollo de esta investigación. 
Figura 10. Número de citas por autor

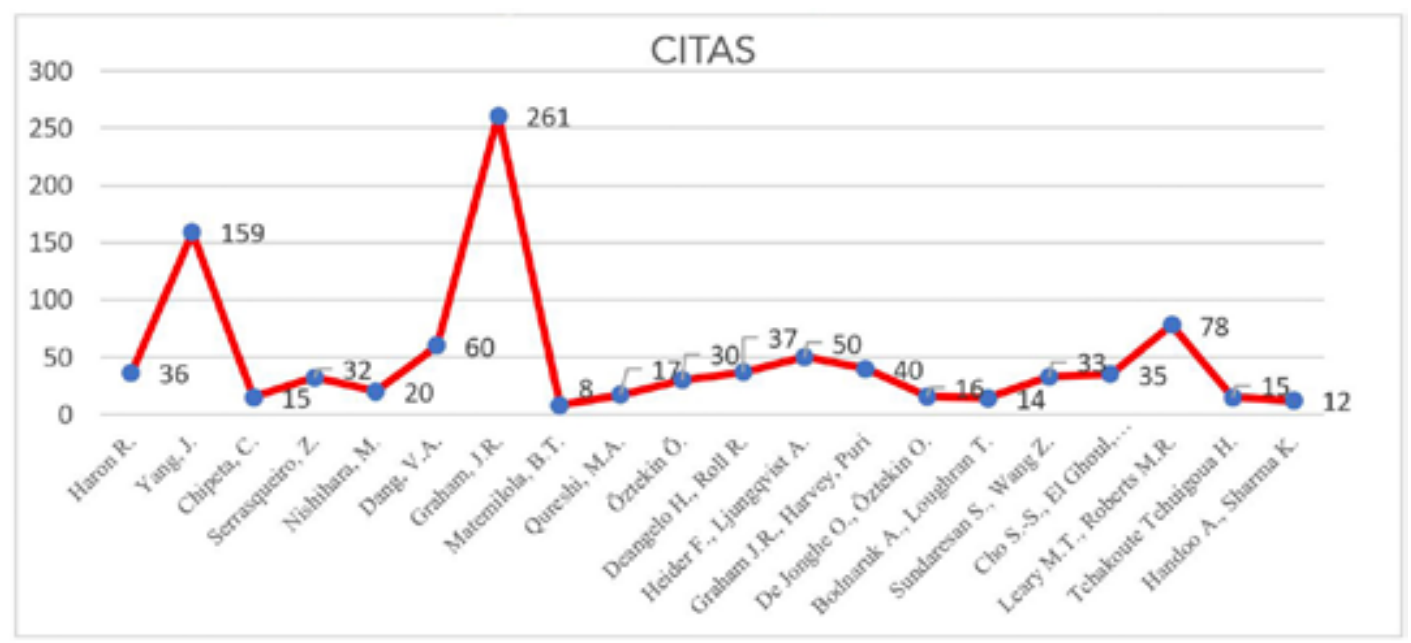

Fuente: Scopus (www.scopus.com)

Elaborado por: Gabriela González.

Los documentos de los autores que mayor fuente de consulta registran son: Graham 261 visitas, Yang, J. posee 159 consultas, Leary M.T., Roberts M.R. un total de 78 concurrencias, Heider F., Ljungqvist A., 50 registros de entradas, Haron R. registró 36 visitas, entre otros autores lo que evidencia un total de 5.248 citas desde el año 2013 al 2018. Este valor es mínimo en relación al lapso de tiempo de cinco años. 
Figura 11. Documentos por país

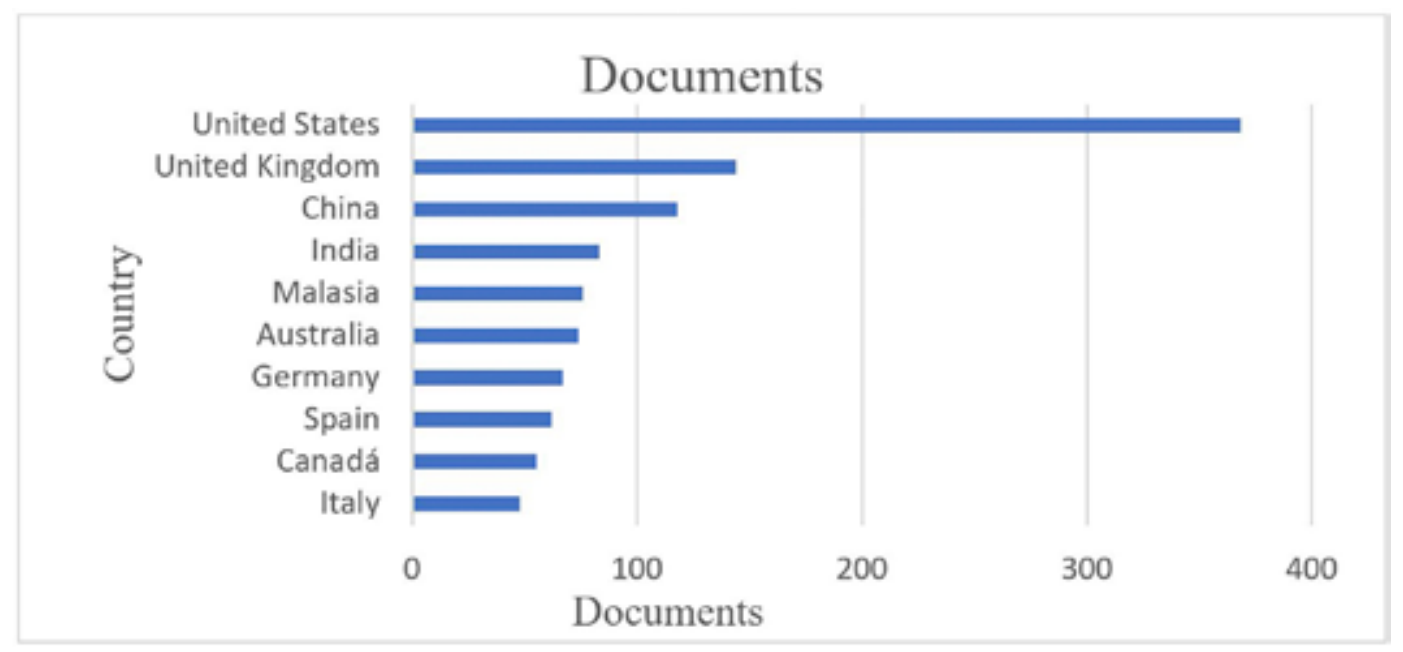

Fuente: Scopus (www.scopus.com)

Elaborado por: Gabriela González.

Se determinó que el país con mayor número de publicaciones es Estados Unidos con 368, United Kingdom 144 documentos, China 118, India 83, Malasia 76, Australia 74, Germany 67, Spain 62, Canadá 55 e Italia con 48 publicaciones relacionadas a la estructura de capital. 


\section{Análisis cualitativo}

\section{Extraída la información de Scopus de los datos \\ asociados a Source List y Bibliographical información \\ se exportó e ingresó los documentos en el programa \\ ATLAS.ti. V8. Anexo 1. A continuación, se describen los \\ pasos hasta la obtención de resultados.}

1. Importación de documentos.

2. Los documentos se cargan en ATLAS.ti. (Carga exitosa) Anexo 2

3. Introducción de documentos para procesamiento. Anexo 3

4. Citas y códigos documentos. Anexo 4

5. Se establece el tipo de relación (link) que se crea correcto, a base de las ideas obtenidas y su relación. Anexo 8

Se empieza a citar y agregar códigos, para esto, se buscan ideas principales sobre el tema investigado; se debe cuidar su similitud para que la red final del análisis permita generar conexiones sin dejar códigos sueltos. Para representar cada idea se selecciona el contexto, se genera como cita y, posteriormente, se le asigna un código como causa o consecuencia de la idea. Ver anexo 4. Una vez generado las ideas principales con sus respectivos códigos, Anexo 7, se exporta la tabla a Excel y se obtiene los siguientes resultados. 
Tabla 2. Códigos generados a partir de las ideas principales

\section{Códigos}

América Latina

Análisis en empresas del sector industrial

Cambio de moneda

Crisis debido a la devaluación

Decisiones financieras

Nivel de apalancamiento

Países desarrollados

Variables que afectan se modifican

Fuente: Scopus (www.scopus.com)

Elaborado por: Gabriela González.

Se establece el tipo de relación (link) que se crea correcto, a base de las ideas obtenidas y su relación, la cual, significa la correlación de códigos dependiente de la experiencia del usuario; finalmente, se obtiene los resultados del análisis cualitativo. Abrimos la red que se generó para editarla si es necesario, como ejemplo se observa en el Anexo 9. 


\section{Argumentación crítica de los resultados}

Sobre el análisis cualitativo se llegó a las siguientes conclusiones:

Figura 12. Análisis cualitativo

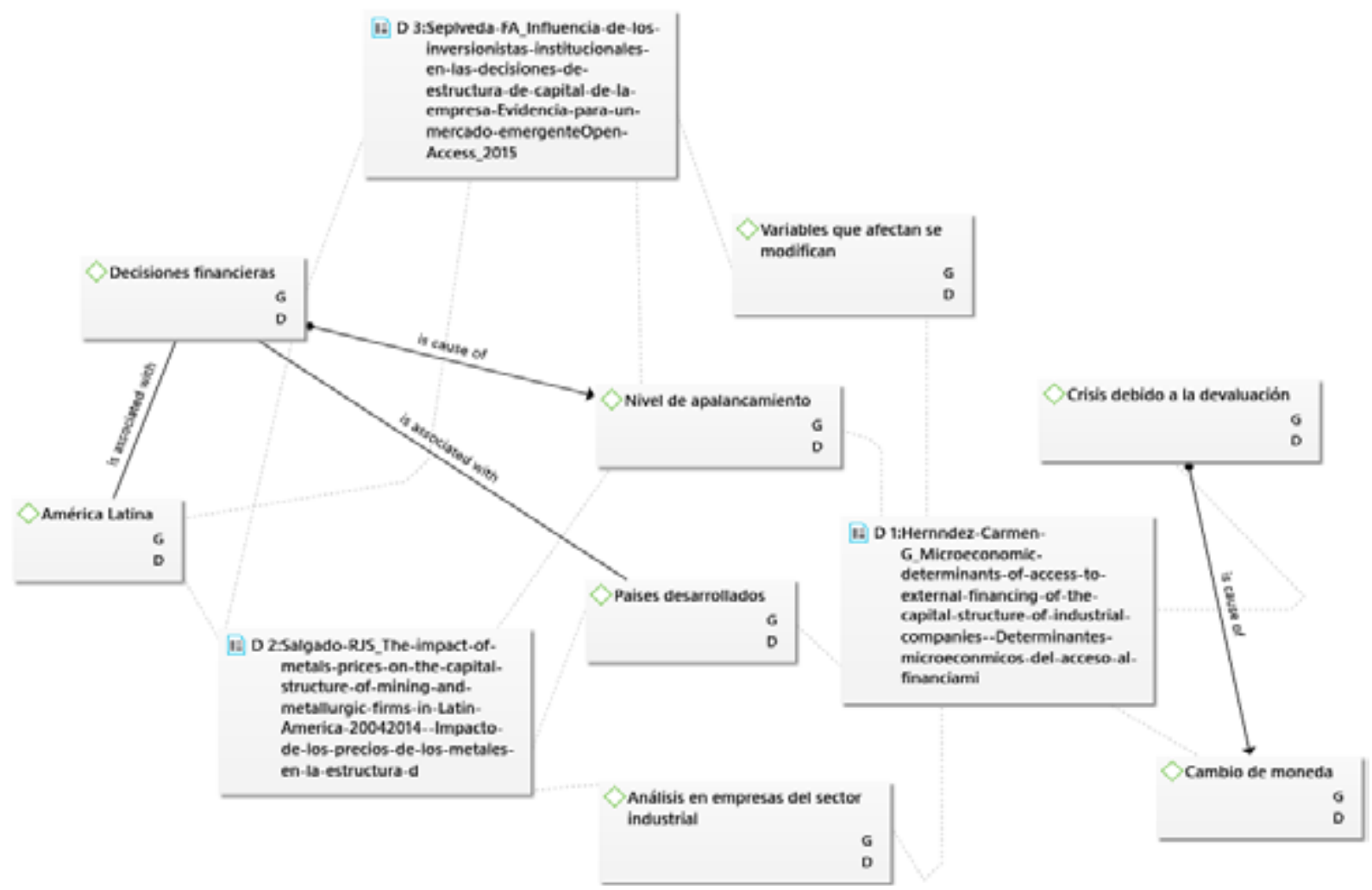

Fuente: Scopus (www.scopus.com)

Elaborado por: Gabriela González. 
En la unidad heurística estructura de capital, encontramos ciertos factores comunes dentro de las investigaciones que se orientan sobre la toma de decisiones financieras y sobre el nivel de endeudamiento o apalancamiento. Las decisiones financieras están asociadas con el territorio, es decir, en América Latina se tienen unos resultados $y$, en cambio, en otros países los resultados son diferentes; a su vez, estas decisiones financieras son causa del nivel de endeudamiento o apalancamiento.

También tenemos que la crisis de la estructura de capital se relaciona con la devaluación, la cual, es una causa del cambio de moneda extranjera. Como factores aislados hemos encontrado que, para el análisis de la estructura de capital, se lleva a cabo más comúnmente en empresas industriales y que las variables que afectan a la estructura de capital, varían con el pasar de los años. Luego del resultado cuantitativo se ha llevado a cabo la generación de una tabla de relación entre los códigos mencionados y los documentos fuente, para poder visualizar el número de veces que aparece un código en las citaciones de los documentos. En este análisis se puede observar que el número de veces que aparece un código, concuerda con el análisis cualitativo que se hizo mediante la red generada sobre las citaciones y códigos de los documentos. Ver anexo 10. 
Tabla 3. Relación entre códigos y documentos: Factor de impacto

\begin{tabular}{|c|c|c|c|c|}
\hline Códigos & $\begin{array}{l}\text { D 1: Hernndez-Carmen- } \\
\text { G_Microeconomic- } \\
\text { determinants-of-access-to- } \\
\text { external-financing-of-the- } \\
\text { capital-s tructure-of- } \\
\text { indus trial-companies-- } \\
\text { Determinantes- } \\
\text { microeconmicos-del-acceso- } \\
\text { al-financiami }\end{array}$ & \begin{tabular}{|c|} 
D 2: Salgado-RJS_The-impact- \\
of-metals-prices-on-the-capital- \\
structure-of-mining-and- \\
metallurgic-firms-in-Latin- \\
America-20042014--Impacto-de- \\
los-precios-de-los-metales-en-la- \\
estructura-d
\end{tabular} & $\begin{array}{c}\text { D 3: Seplveda-FA_Influencia-de- } \\
\text { los-inversionistas- } \\
\text { institucionales-en-las- } \\
\text { decisiones-de-es tructura-de- } \\
\text { capital-de-la-empresa-Evidencia- } \\
\text { para-un-mercado- } \\
\text { emergenteOpen-Access_2015 }\end{array}$ & $\begin{array}{l}\text { Total } \\
\text { por } \\
\text { código }\end{array}$ \\
\hline América Latina & 0 & 2 & 2 & 4 \\
\hline $\begin{array}{l}\text { Anális is en empres as del } \\
\text { sector indus trial }\end{array}$ & 6 & 3 & 0 & 9 \\
\hline Cambio de moneda & 3 & 0 & 0 & 3 \\
\hline $\begin{array}{l}\begin{array}{l}\text { Crisis debido a la } \\
\text { devaluación }\end{array} \\
\end{array}$ & 1 & 0 & 0 & 1 \\
\hline Decisiones financieras & 0 & 1 & 1 & 2 \\
\hline Nivel de apalancamiento & 4 & 3 & 3 & 10 \\
\hline Pais es des arrollados & 1 & 1 & 0 & 2 \\
\hline $\begin{array}{l}\text { Variables que afectan se } \\
\text { modifican }\end{array}$ & 1 & 0 & 1 & 2 \\
\hline Total por documento & 16 & 10 & 7 & 33 \\
\hline
\end{tabular}

Fuente: Scopus (www.scopus.com)

Elaborado por: Gabriela González. 
Se obtuvo una total de 33 códigos y documentos, con mayor número el índice de impacto se encuentra en documentos de Nivel de apalancamiento (10 códigos) Análisis en empresas del sector industrial (9 códigos), América latina (4 códigos) y Cambio de moneda (3 códigos).

Se concluye que, el aporte a la ciencia y a la comunidad académica es, sin duda, el parámetro más importante sobre la productividad de los artículos e investigaciones científicas, ya que plantean un marco de referencia o punto de partida para nuevas investigaciones, es por esto que se evita tener redundancia, es decir varias investigaciones sobre el mismo tema; $y$, por último, ayuda a los investigadores a retomar estos artículos y basándose en sus conclusiones, realizar un estudio más complejo.

Para el estudio bibliométrico algo importante es saber qué fuente resulta mejor para la consulta de información, ya que con esto reducimos la probabilidad de encontrar información errónea; esto se lo realiza en base a los indicadores de impacto, que en este caso resultó ser en mayor porcentaje el de Scopus.

Una vez que se tiene una plataforma web para la búsqueda de datos, es necesario saber de una manera muy específica qué es lo que se va a buscar; para esto hacemos uso de los descriptores que se relacionan directamente con los artículos o revistas, pero, no con su contenido en sí, sino con sus filtros, es decir: título, etiquetas (tags), abstract, tema de área, etc.; de esta manera, la búsqueda de información se la hace de manera ordenada, obteniendo resultados precisos del tema consultado, en este caso se usó como título: capital structure, y como área de estudio: Economics, econometrics and Finance, Business, Management and Accounting, Social Sciences y Decision Sciences.

Finalmente, el conocimiento teórico - científico sobre la estructura de capital facilitó la comprensión sobre las ventajas y desventajas que puede causar sobre la situación de cada empresa, dejando evidente que cada organización selecciona su propia estructura de capital según sus conveniencias y a base de los factores internos y externos que condicionan las decisiones de administradores y accionistas. 


\section{REFERENCIAS BIBLIOGRÁFICAS}

Akerlof, G. (1970). The Quarterly Journal of Economics, 84, 488. http://www.scielo.org.bo/ scielo.php?script=sci_arttext\&pid=S2518-44312014000100004

Alperin, J., Babini, D. (2014). Redalyc una plataforma para la visibilidad de la producción científica del mundo, publicada en revistas de Iberoamérica. CLASCO, Editor. https://www. researchgate.net/publication/272419278_Redalyc_una_plataforma_para_la_visibilidad_de_la_produccion_cientifica_del_mundo_publicada_en_revistas_de_lberoamerica.

Alvarado, R. (2011). http://eprints.rclis.org/17674/1/Urbizagastegui-Art-v40n2-2011.pdf.

Amézquita, J., Martínez, D., Martínez, J., \& Maza, F. (2011). http://190.242.62.234:8080/jspui/ bitstream/11227/245/1/modulo\%20\%20CTS\%20No4-cienciometria.pdf.

Aragón, I. (11 de 1995). http://biblioteca.ucm.es/tesis/19911996/X/3/X3023101.pdf.

Ardanuy, J. (2012). Breve introducción de la bibliometría http://diposit.ub.edu/dspace/bitstream/2445/30962/1/breve\%20introduccion\%20bibliometria.pdf

Argudo \& Becerril. (2014). http://www.redalyc.org/pdf/601/60144179001.pdf.

Arrow, K. (1971). The Theory of discrimination. Industrial Relations Section. Princeton University, 30, 1. http://www.scielo.org.bo/scielo.php?script=sci_arttext\&pid=S2518-44312014000100004

Bernal, D. (2013). Teorías, enfoques y aplicaciones en las Ciencias Sociales. Revista Científica. http://132.248.9.34/hevila/Revistacientificateoriasenfoquesyaplicacionesenlascienciassociales/2013/no11/7.pdf.

Cabrera, B., Rico, P. (2015). E. d. Aplicada, Editor.

http://www.redalyc.org/pdf/301/30141391009.pdf.

Cardoso, P. (2017). Ecuador tiene solo 3 revistas científicas de impacto. EcuadorUniversitario. http://ecuadoruniversitario.com/de-instituciones-del-estado/senescyt/ecuador-tiene-solo-3-revistas-cientificas-de-impacto/.

Chaves, N., Rueda, E., Sousa, J., Siqueira, J., Straddiotto, J., Nepomuceno, E., \& Holanda, L. (2016). Revista Invenio vol 19, núm 7. http://www.redalyc.org/pdf/877/87747436003. pdf

Contreras, N. \& Diaz, E. (01 de 04 de 2015). E. P. Contabilidad, Editor, \& U. P. Unión, Productor. file:///C:/Users/adm/Downloads/824-1067-1-PB.pdf.

Coronel,C. (12 de 2015). (E. S. Litoral, Ed.) http://www.dspace.espol.edu.ec/xmlui/bitstream/ handle/123456789/34587/D-CSH154.pdf?sequence=-1\&isAllowed=y. 
Correa, D. (2013). http://www.tdx.cat/bitstream/handle/10803/9314/Tesi_Marc_Correa.pd$f$ ?sequence $=1$ \&isAllowed $=y$.

Dávila, D. M. (2015). Bilbiometría. Conceptos y utilidades. http://www.bibliotecaminsal.cl/ wp/wp-content/uploads/2015/07/Bibliometria-conceptos-y-utilidades.pdf.

Dávila, G. (2006). Revista Redalyc vol 12, núm 1. http://www.redalyc.org/pdf/761/76109911. pdf

Dittmar, B. (2001). http://business.illinois.edu/finance/papers/2001/dittmar.pdf.

Ebrain, E. (2016). https://www.sciencedirect.com/science/article/pii/S2007505717301709.

Escobedo, L. (2013). Evolución de la Teoría sobre la estructura financiera óptima en las empresas . http://xn--caribea-9za.eumed.net/wp-content/uploads/palancamiento.pdf

Escobedo, L. (06 de 2016). La estructura financiera óptima bajo un enfoque de ciencia, tecnología y sociedad. (U. d. Habana, Ed.) Scielo, 10(1). http://scielo.sld.cu/scielo. php?script=sci_arttext\&pid=S2073-60612016000100007

Escorcia, T. (2008). http://www.javeriana.edu.co/biblos/tesis/ciencias/tesis209.pdf.

Escorcia, T. (2008). Repositorio Universidad Javeriana. http://www.javeriana.edu.co/biblos/ tesis/ciencias/tesis209.pdf

Escuela Politécnica Nacional. (2017). http://www.epn.edu.ec/la-revista-politecnica-paso-a-ser-catalogo-de-latindex/.

Esparza, J. (2012). Estructura del Capital . http://web.uqroo.mx/archivos/jlesparza/acpef140/3.3a\%20Estructura\%20capital.pdf

Fernández, M. (2017). (U. Jaume, Editor). http://repositori.uji.es/xmlui/bitstream/handle/10234/171803/TFM_2017_FernandezFiant_MariaAgustina.pdf?sequence=1.

Filipo. (2013). Bibliometría. Importancia de los indicadores bibliométricos. http://www.ricyt.org/manuales/doc_view/113-bibliometria-importancia-de-los-indicadores-bibliometricos.

Filippo. (2013). Bibliometría: Importancia de los indicadores bibliométricos. https://www. researchgate.net/publication/263653448_Bibliometria_Importancia_de_los_indicadores_bibliometricos.

Filippo, D., \& Fernández, M. T. (2012). Bibliometría: Importancia de los indicadores bibliométricos. http://www.ricyt.org/manuales/doc_view/113-bibliometria-importancia-de-los-indicadores-bibliometricos

Fillipo, D. d. (2002). Bibliometría. Importancia de los indicadores bibliométricos. https://www. researchgate.net/publication/263653448_Bibliometria_Importancia_de_los_indicadores_bibliometricos

González, J. (1997). https://www.aeped.es/sites/default/files/anales/47-3-3.pdf.

Gorbea, S. (10 de 2016). http://www.scielo.org.mx/pdf/ib/v30n70/0187-358X-ib-30-70-00011. pdf. 
Hackbarth, D., Hennessy, C., Leland, H. (2011). Can the Trade-off Theory Explain Debt Structure? The Review of Financial Studies, 20.

Hérubel, J. (1999). Historical Bibliometrics: Its Purpose and Significance to the History of Disciplines. Libraries and Culture.

Juárez, P. (2016). http://www.upch.edu.pe/vrinve/dugic/revistas/index.php/RENH/article/ viewFile/2864/2731.

Kim, E. (1978). A mean- variance theory of optimal capital structure and corporate debt capacity. Journal of Finance, 32(1).

Kraus, A. \& Litzanberger. (1973). A State-Preference Model of Optimal Financial Leverage. Journal of Finance.

Markowitz, H. (1952). Portafolio Selection. Journal of Finance, 7, 77 a 90.

Martín, A. (2018). http://bibliosjd.org/2018/03/13/revista-indexada/\#.W4XTK-jOnIU.

Mejía, A. (05 de 09 de 2013). 5(2). Recuperado el 02 de 10 de 2018, de http://www.scielo.org. co/pdf/fype/v5n2/v5n2a08.pdf

Mejía, A. (05 de 09 de 2013). 5(2). Recuperado el 02 de 10 de 2018, de http://www.scielo.org. co/pdf/fype/v5n2/v5n2a08.pdf

Miguel, S. (03 de 06 de 2011). http://www.redalyc.org/html/1790/179022554006/.

Modigliani, F. \& Miller, M. (1958). The Cost of Capital. American Economic, 48, 261 a a292.

Mongrut, S., Fuenzalida, D. Pezo, G., Teply, Z. (10 de 09 de 2010). http://www.scielo.org.co/ pdf/cadm/v23n41/v23n41a08.pdf.

Moreira \& Rodríguez. (01 de 06 de 2006). file://C:/Users/adm/Downloads/824-1067-1-PB. pdf.

Morocho, J. (2018). Análisis bibliométrico de la producción científica en el sistema de educación superior ecuatoriano. http://bibdigital.epn.edu.ec/bitstream/15000/19202/1/ CD-8576.pdf

Myers, S. (1984). The Capital Structure Puzzle. The Journal of Finance.

Myers, Stewart y Majluf. (1984). (J. O. Economics, Productor) http://ta.ba.ttu.edu/cjun/class/ FIN6331_2009/articles/Myers\%20and\%20Majluf\%20\%281984\%29.pdf.

Narin, F. (1976). Evaluative Bibliometrics: The use of publication and citation analysis. New Jersey: Computer Horizons Cherry Hill.

Orozco , W., \& Quijano , L. (2014). Repositorio Universidad de San Francisco. http://repositorio.usfq.edu.ec/bitstream/23000/3839/1/112559.pdf

Patrón , C., López, J., Piovesan, S., \& Demaría, B. (2014). http://www.scielo.edu.uy/pdf/ode/ v16n23/v16n23a05.pdf.

Quillis, V. (2015). http://www.bvsspa.es/profesionales/sites/default/files/documentos/BIBLIOMETRIASALUDINVESTIGA.pdf 
Repositorio Universidad Católica de Chile . (2018). http://guiastematicas.bibliotecas.uc.cl/ editoresUC/editoresuc/postular_scopus

Revelo-Oña, R. M. (20 de 12 de 2017). Determinación de la estructura óptima de capital del sector comercial al por mayor. Ecuador período 2014 - 2015. (ResearchGate, Ed.) Digital Publisher(4), 56-66. https://www.researchgate.net/publication/322131524_Determinacion_de_la_estructura_optima_de_capital_del_sector_comercial_al_por_mayor_Ecuador_periodo_2014_-_2015

Ross, A. (1977). The Bell Journal of Economics, 8(1), 23-40. http://www.scielo.org.bo/scielo. php?script=sci_arttext\&pid=S2518-44312014000100004.

Rueda, C., Villaroel, C., \& Rueda, C. (2005). Recuperado el 10 de 09 de 2018, de file:///C:/ Users/SafeComputer/Downloads/564-979-1-SM.pdf

Salazar, S, Bautista, I. (30 de 12 de 2017). http://www.redalyc.org/pdf/346/34653156008.pdf.

Sanz, J. (2014). Estudio bibliométrico de la producción científica publicada por la Revista Panamericana de Salud Pública. https://www.scielosp.org/article/rpsp/2014.v35n2/8188/

SClmago Institutions Rankings (SIR). (2018). https://www.uv.es/uvweb/servicio-analisis-planificacion/es/rankings/observatorio-rankings-/-scimago-institutions-rankings-1285868425862.html.

Shyam, L. y Myers, S. (1994). (W. Paper, Editor, \& MIT Sloan School of Management) http:// dspace.mit.edu/bitstream/handle/1721.1/49224/testingstatictra00shya.pdf?sequence $=1$

Spence, M. (1973). Job Market Signaling. The Quarterly Journal of Economics, 355. http:// www.scielo.org.bo/scielo.php?script=sci_arttext\&pid=S2518-44312014000100004

Vallejos, R. (2005). http://www.revistasbolivianas.org.bo/scielo.php?pid=S2313-0229201400 0100007\&script=sci_arttext.

Vargas, A. (2 de 2014). Estructura de capital óptima en presencia de costos de dificultades financieras. Investigación y Desarrollo, 1(14). http://www.scielo.org.bo/scielo. php?script=sci_arttext\&pid=S2518-44312014000100004

Vargas, A. (2014). Estructura de capital óptima en presencia de costos de dificultades financieras. (U. P. Boliviana, Ed.) SciELO, 1(14). Recuperado el 11 de 12 de 2018, de http:// www.scielo.org.bo/scielo.php?script=sci_arttext\&pid=S2518-44312014000100004

Velasco, B., \& Eiros, J. (2012). La utilización de los indicadores bibliométricos para evaluar la actividad investigadora. Revista Aula Abierta vol 40, Núm 2. file:///C:/Users/SafeComputer/Downloads/Dialnet-LaUtilizacionDeLosIndicadoresBibliometricosParaEva-3920967.pdf

Veronesi, P. (2000). How Does Information Quality Affect Stock Returns? Journal of Finance, 55(2). Recuperado el 05 de 10 de 2018, de http://www.scielo.org.bo/scielo. php?script=sci_arttext\&pid=S2518-44312014000100004 
Villaroel, K. (10 de 2014). (R. d. Scientia, Editor) http://www.revistasbolivianas.org.bo/scielo. php?pid=S2313-02292014000100007\&script=sci_arttext.

Zambrano \& Acuña. (07 de 2011). Estructura de Capital. Evolución Teórica. file:///C:/Users/ adm/Downloads/Dialnet-EstructuraDeCapitalEvolucionTeorica-3815888\%20(2).pdf. (U. L. Colombia, Productor)

Zambrano \& Acuña. (12 de 2011). file:///C:/Users/adm/Downloads/Dialnet-EstructuraDeCapitalEvolucionTeorica-3815888.pdf.

Zambrano \& Acuña. (2013). https://www.dspace.espol.edu.ec/retrieve/129011/D-CD293.pdf. Apuntes del CENES, 32. 


\section{ANEXOS}

Anexo 1. Nuevo proyecto ATLAS.ti con datos extraídos de Scopus

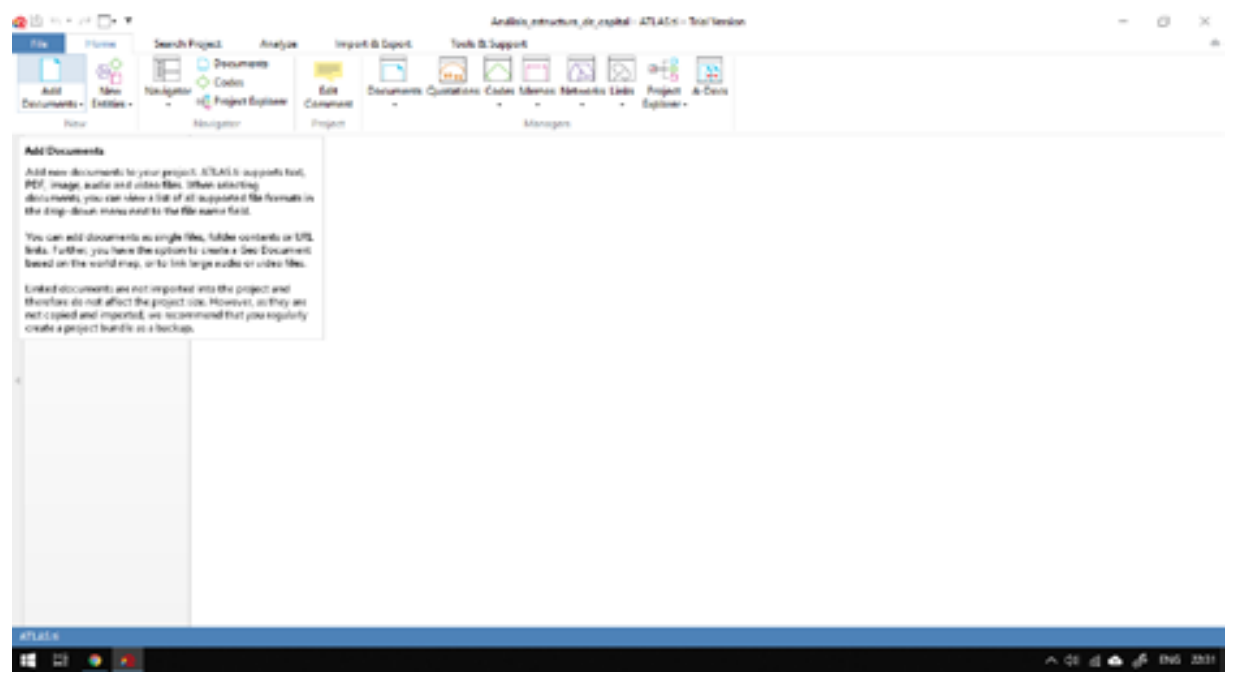

Anexo 2. Carga de documentos en ATLAS.ti

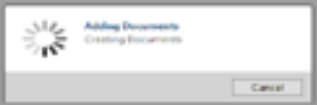




\section{Anexo 3. Importación de documentos para procesamiento}

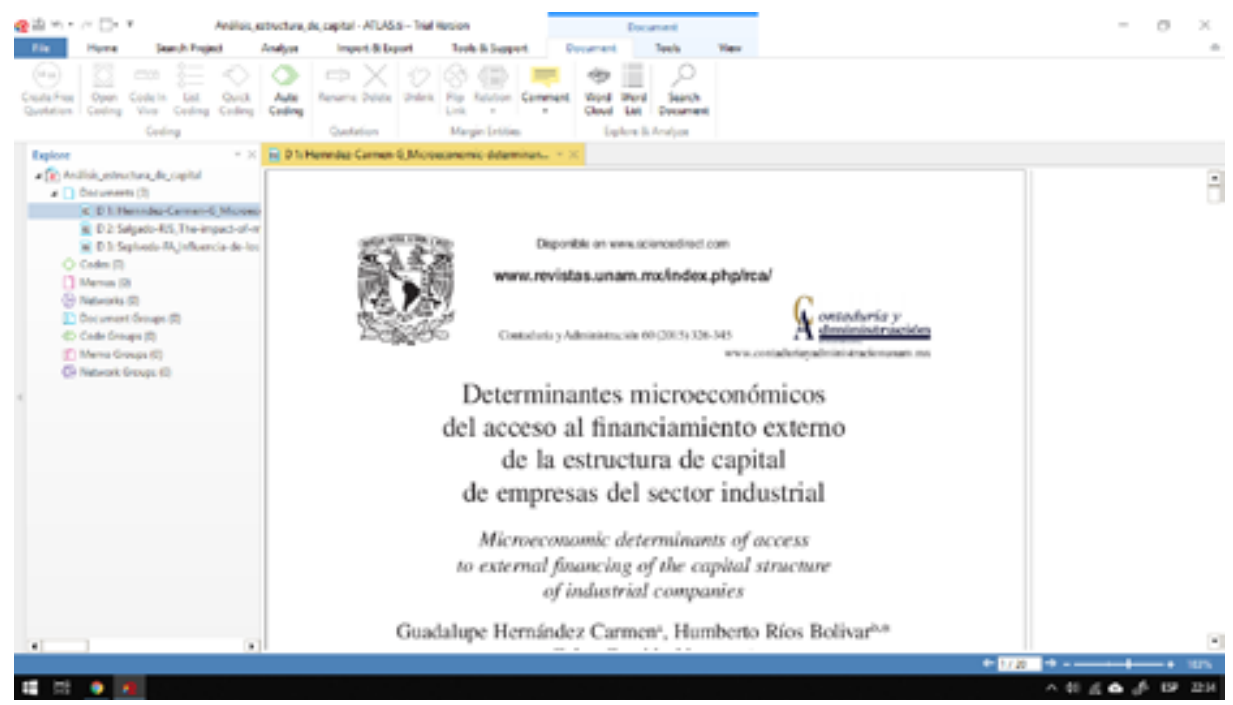

Anexo 4. Citas y códigos documento 1

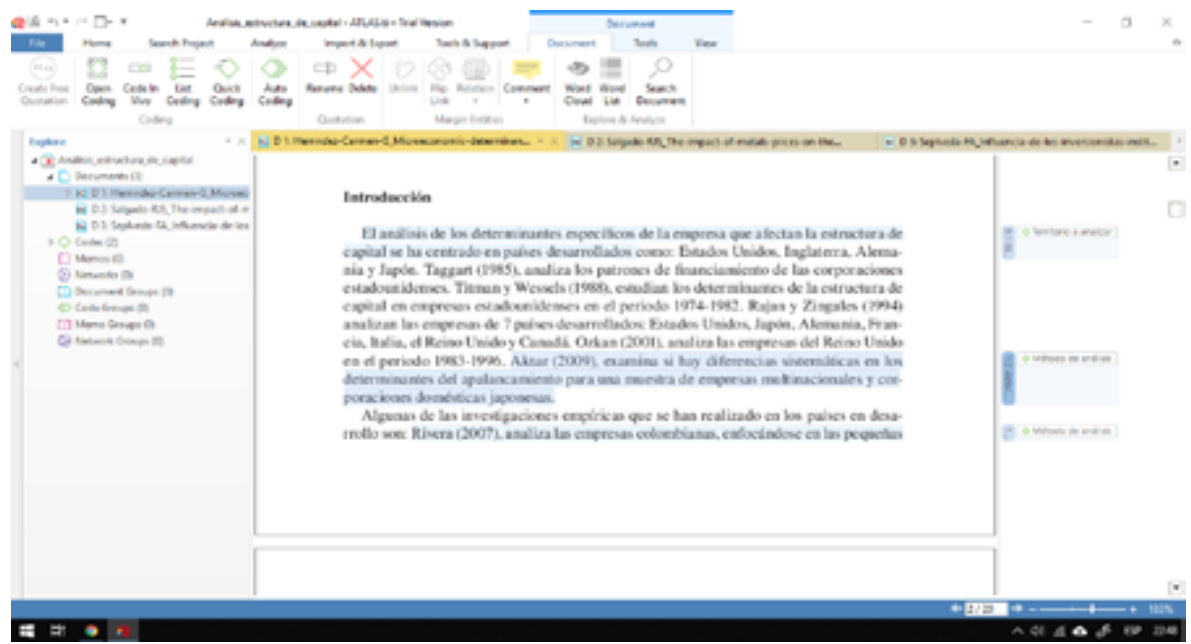




\section{Anexo 5. Citas y códigos documento 2}

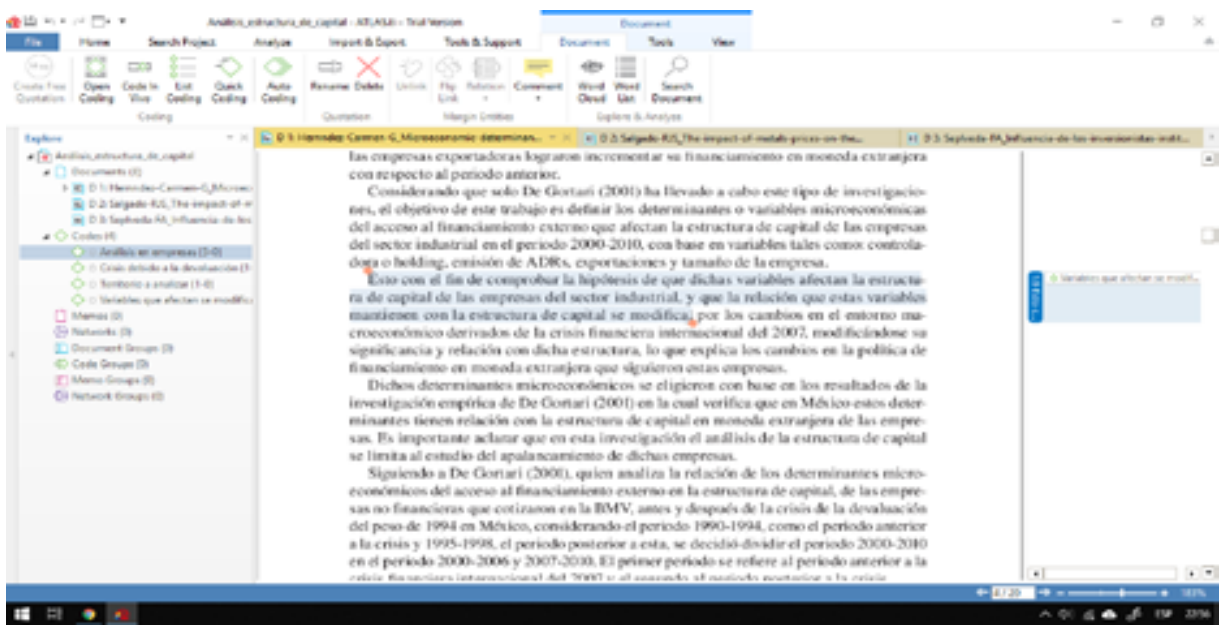

\section{Anexo 6. Citas y códigos documento 3}

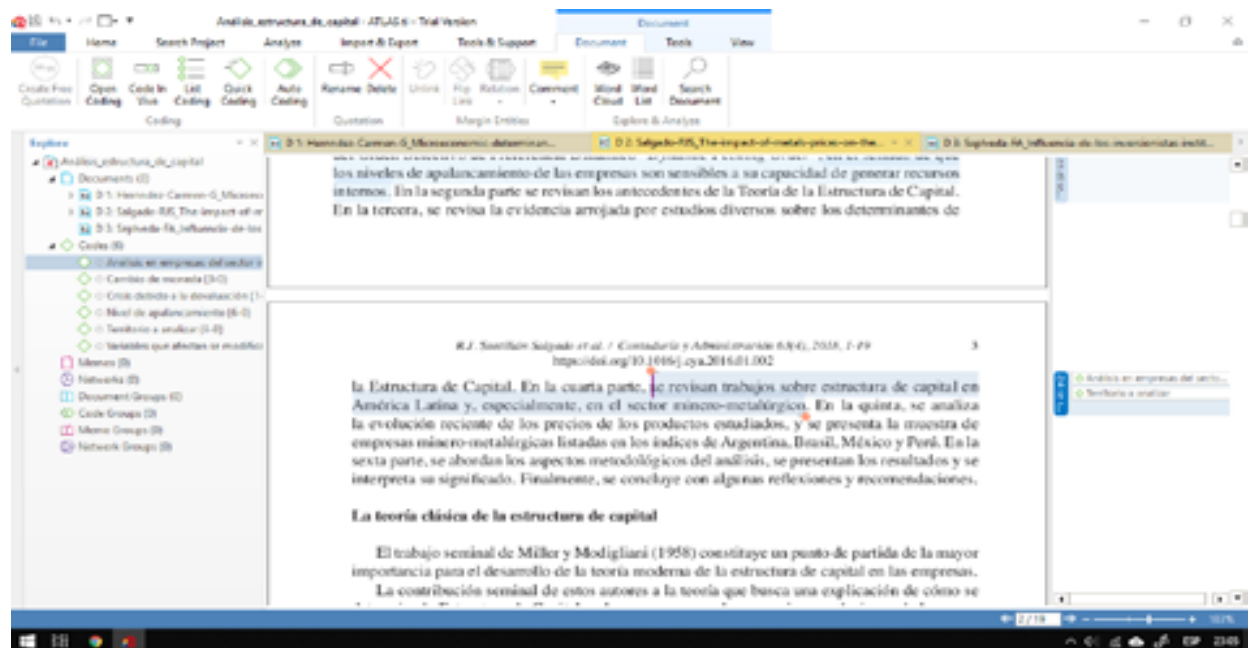


Anexo 7. Generación ideas principales y códigos

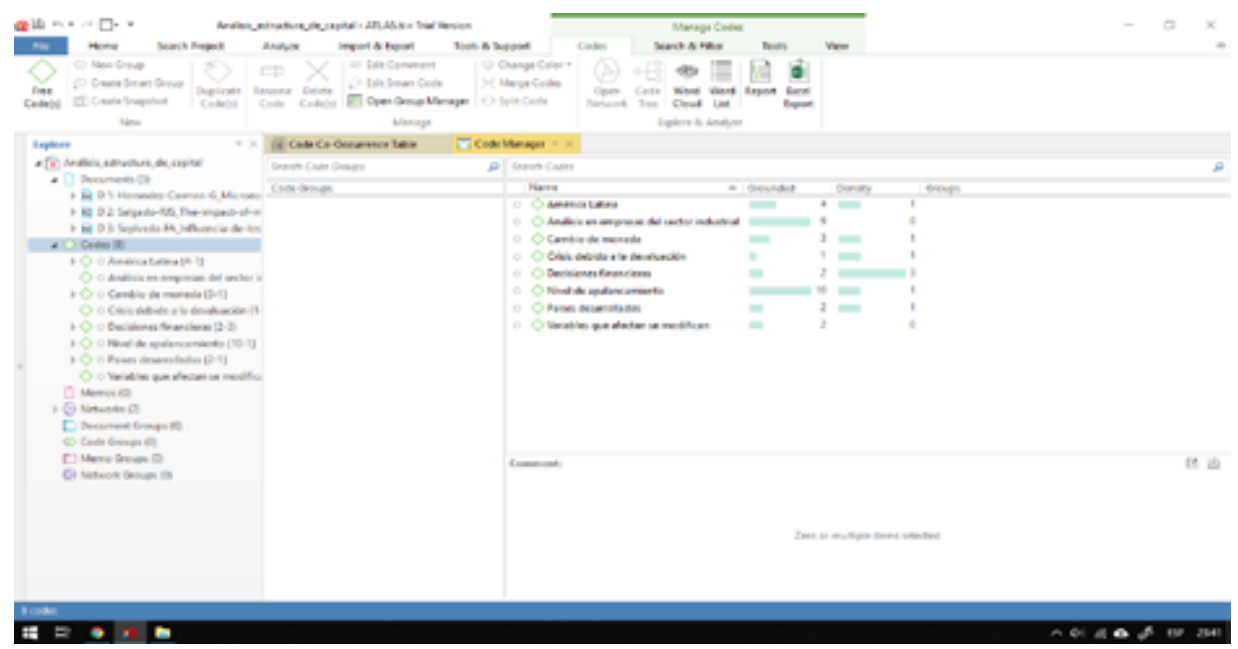

Anexo 8. Establecimiento de tipo de relación en base a ideas

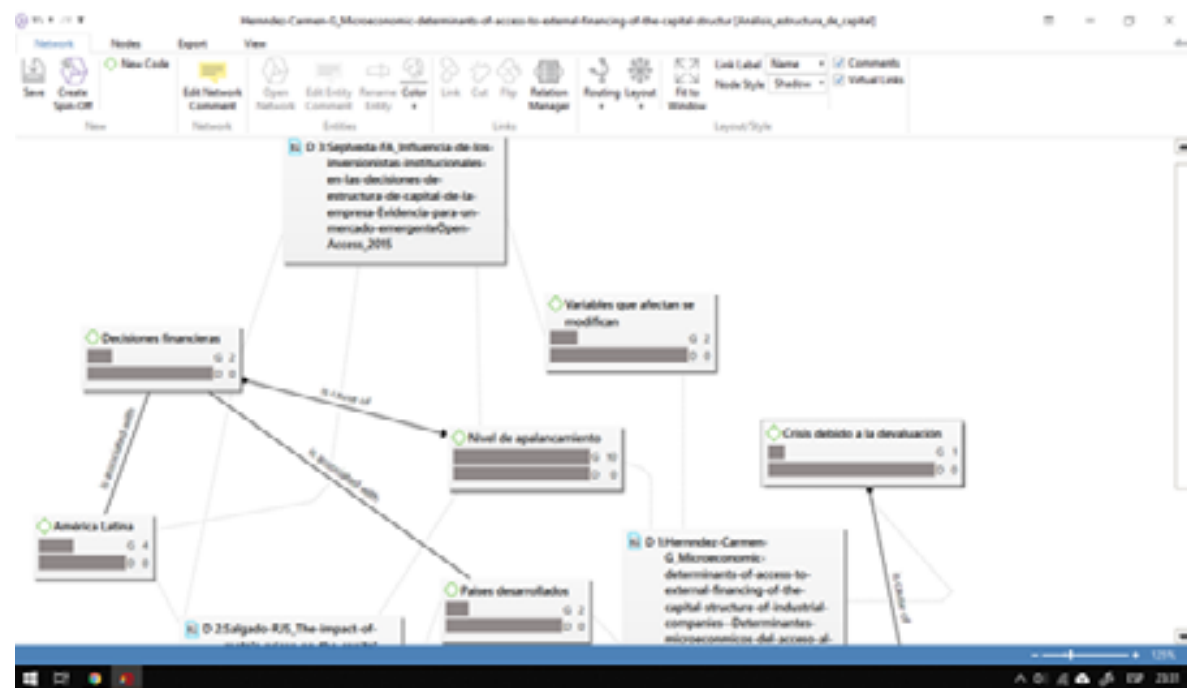


Anexo 9. Generación de red

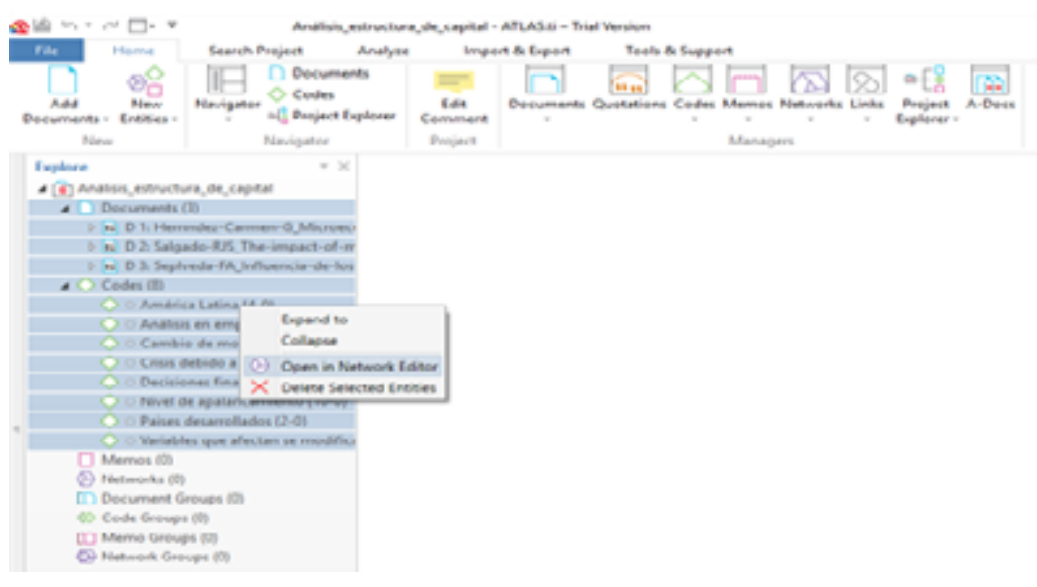

Anexo 10. Citaciones y códigos de documentos

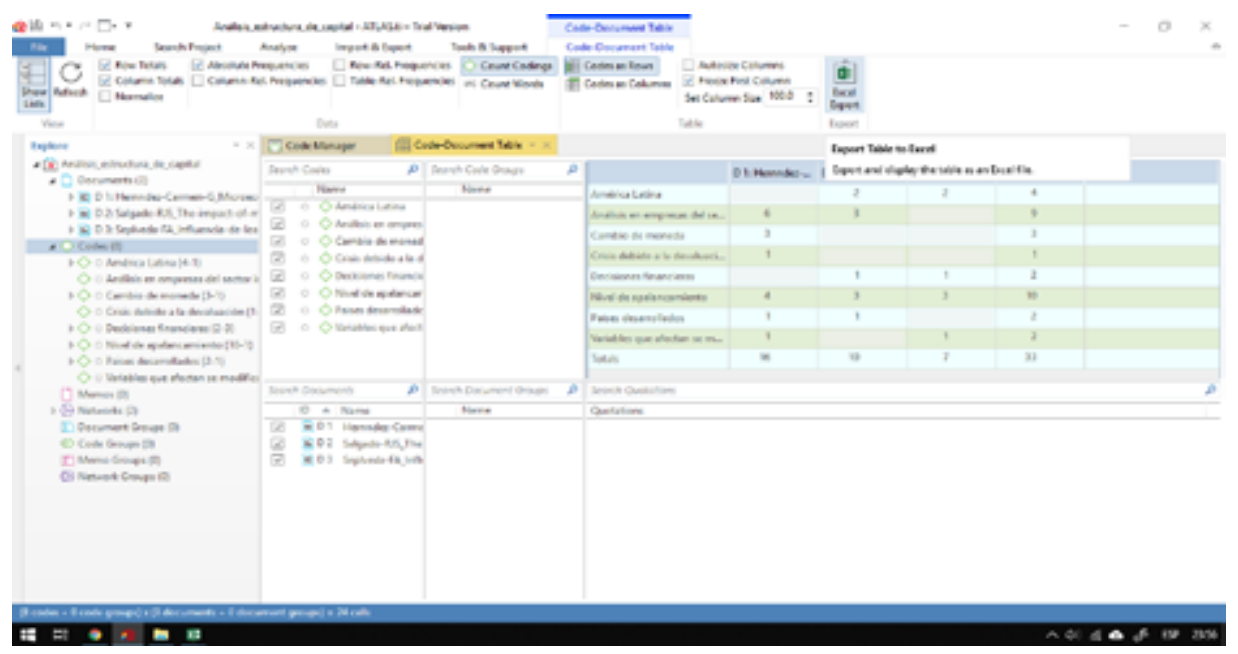

